\title{
DISCRETE-TIME AND SAMPLED-DATA LOW-GAIN CONTROL OF INFINITE-DIMENSIONAL LINEAR SYSTEMS IN THE PRESENCE OF INPUT HYSTERESIS*
}

\author{
H. LOGEMANN ${ }^{\dagger}$ AND A. D. MAWBY ${ }^{\dagger \dagger}$
}

\begin{abstract}
We introduce a general class of causal dynamic discrete-time nonlinearities which have certain monotonicity and Lipschitz continuity properties. In particular, the discretizations of a large class of continuous-time hysteresis operators obtained by applying the standard sampling and hold operations belong to this class. It is shown that closing the loop around a power-stable, linear, infinite-dimensional, discrete-time, single-input, single-output system, subject to an input nonlinearity from the class under consideration and compensated by a discrete-time integral controller, guarantees asymptotic tracking of constant reference signals, provided that (a) the positive integrator gain is sufficiently small and (b) the reference value is feasible in a very natural sense. We apply this result in the development of sampled-data low-gain integral control for exponentially stable, regular, linear, infinite-dimensional, continuous-time systems subject to input hysteresis.
\end{abstract}

Key words. continuous-time regular infinite-dimensional systems, discrete-time infinite-dimensional systems, hysteresis nonlinearities, integral control, robust tracking, sampled-data control

AMS subject classifications. 47H30, 47J40, 93C10, 93C20, 93C25, 93C55, 93C57, 93D10

PII. S0363012901385770

1. Introduction. The present paper extends the line of work on low-gain integral control of infinite-dimensional linear systems subject to input nonlinearities initiated by the recent papers [9], [11], [14], [15]. Underpinning these contributions are generalizations of the well-known principle (see, for example, [4], [17], [18], and [22]) that closing the loop around a stable, linear, continuous-time, single-input, single-output plant, with transfer function $\mathbf{G}^{c}(s)$ compensated by a pure integral controller $k / s$, will result in a stable closed-loop system that achieves asymptotic tracking of arbitrary constant reference signals, provided that $|k|$ is sufficiently small and $\mathbf{G}^{c}(0) k>0 .{ }^{1} \mathrm{In}$ particular, Logemann and Mawby [11] have shown that the above principle remains true for exponentially stable, regular, linear, infinite-dimensional, continuous-time, single-input, single-output systems subject to input hysteresis belonging to a certain class $\mathcal{C}(\lambda)$ of hysteresis operators, provided the reference value $r$ is feasible in a natural sense. The class $\mathcal{C}(\lambda)$ consists of hysteresis operators which, among other conditions, satisfy a certain Lipschitz condition with Lipschitz constant $\lambda>0$. We emphasize that $\mathfrak{C}(\lambda)$ encompasses a large number of hysteresis nonlinearities important in applications such as relay, backlash, elastic-plastic, and Prandtl hysteresis.

In this paper, we provide discrete-time and sampled-data analogues of the continuous-time results in [11]. More precisely, the contribution of the present work is twofold.

*Received by the editors March 2, 2001; accepted for publication (in revised form) October 8, 2001; published electronically April 2, 2002. This work was supported in part by the UK EPSRC Council (grant GR/L78086).

http://www.siam.org/journals/sicon/41-1/38577.html

${ }^{\dagger}$ Department of Mathematical Sciences, University of Bath, Bath BA2 7AY, UK (hl@maths. bath.ac.uk).

${ }^{\ddagger}$ Current address: Systems Engineering and Assessment, P.O. Box 800, Bath BA3 6TB, UK (adm@sea.co.uk).

${ }^{1}$ Therefore, under the above assumptions on the plant, the problem of tracking constant reference signals reduces to that of tuning the gain parameter $k$. This so-called tuning regulator theory [4] has been successfully applied in process control (see [3], [19]). 


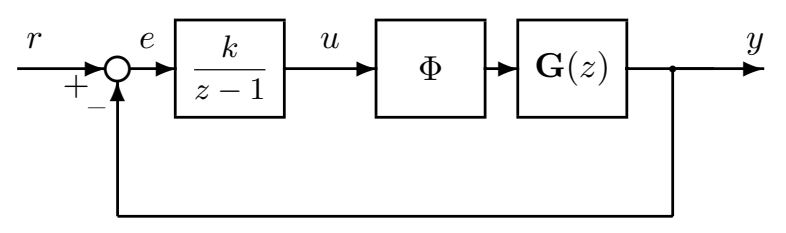

FIG. 1. Low-gain control with input nonlinearity.

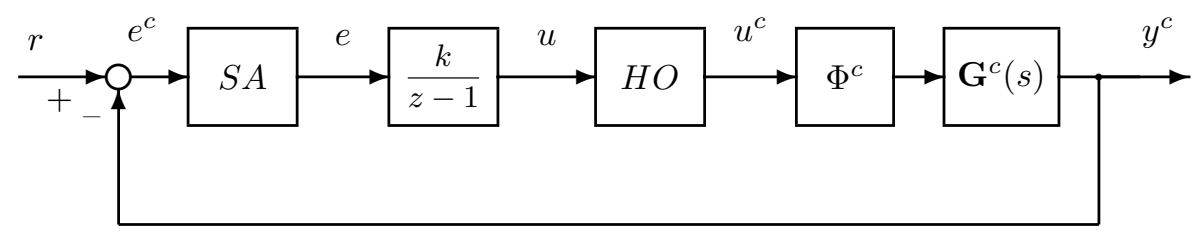

FIG. 2. Sampled-data low-gain control.

(i) We introduce a class $\mathcal{D}(\lambda)$ of causal dynamic discrete-time nonlinearities which have a number of properties, one of them being that a functional Lipschitz condition with Lipschitz constant $\lambda>0$ is satisfied. The class $\mathcal{D}(\lambda)$ contains a large number of discrete-time hysteresis operators, in particular discretizations of continuous-time hysteresis operators in $\mathcal{C}(\lambda)$ obtained by sampling/hold. We derive a discrete-time version of the continuous-time tuning regulator result in [11] by showing that for a power-stable, linear, infinite-dimensional, discrete-time, single-input, single-output plant with transfer function $\mathbf{G}(z)$, subject to a dynamic input nonlinearity $\Phi \in \mathcal{D}(\lambda)$, the output $y(n)$ of the closed-loop system, shown in Figure 1, converges to the reference value $r$ as $n \rightarrow \infty$, provided that $\mathbf{G}(1)>0, r$ is feasible in some natural sense, and $k \in(0, K / \lambda)$, where $K$ is the supremum of the set of all numbers $k>0$ such that

$$
1+k \operatorname{Re} \frac{\mathbf{G}(z)}{z-1} \geq 0 \quad \forall|z|>1 .
$$

(ii) We apply the discrete-time theory in the development of a sampled-data counterpart to the continuous-time low-gain control result in [11] - see Figure 2 -where $H O$ denotes a standard hold operation and $S A$ denotes a sampling operation. In the case of unbounded observation, the latter involves an averaging operation. Specifically, we show that for an exponentially stable, regular, linear, infinite-dimensional, continuous-time, single-input, single-output plant with transfer function $\mathbf{G}^{c}(s)$, subject to a continuous-time dynamic input nonlinearity $\Phi^{c} \in \mathcal{C}(\lambda)$, the output $y^{c}(t)$ of the closed-loop system, shown in Figure 2, converges to the reference value $r$ as $t \rightarrow \infty$, provided that $\mathbf{G}^{c}(0)>0, r$ is feasible in some natural sense, and $k>0$ is sufficiently small. The class of regular, linear, infinite-dimensional, continuoustime systems, introduced by Weiss [25], [26], [27], [28], is rather general. It includes most distributed parameter systems and all time-delay systems (retarded and neutral) which are of interest in applications. With respect to (i), while the structure of the discrete-time analysis parallels that of the continuous-time analysis in [11], there are several points where these analyses differ in an essential manner. With reference to (ii), the sampled-data results constitute the main contribution of the paper. In the derivation of these results, the discrete-time theory plays a central role. 
We mention that there exists a substantial literature on the mathematical theory of hysteresis phenomena; see, for example, Brokate [1], Brokate and Sprekels [2], and Krasnosel'skiı and Pokrovskiu [8]. Of particular importance in a systems and control context is the pioneering work [8]. Our treatment of continuous-time hysteresis operators in section 4 has been strongly influenced by chapter 2 in [2].

The paper is organized as follows. In section 2, we introduce the class $\mathcal{D}(\lambda)$ of dynamic discrete-time nonlinearities, while section 3 contains the low-gain tuning regulator result for discrete-time systems subject to input nonlinearities belonging to $\mathcal{D}(\lambda)$. In section 4 , we introduce the class $\mathcal{C}(\lambda)$ of dynamic continuous-time nonlinearities and establish several important properties enjoyed by nonlinearities in this class. At the end of this section, we discretize continuous-time hysteresis operators and show that the resultant discrete-time operators are contained in the class $\mathcal{D}(\lambda)$. In section 5, the low-gain tracking problem for exponentially stable, regular, linear, infinite-dimensional, continuous-time, single-input, single-output systems with input nonlinearity in $\mathcal{C}(\lambda)$ is solved using sampled-data integral control. In section 6 , we illustrate our results in the context of a simple linear diffusion process subject to input hysteresis: relay as well as backlash nonlinearities are considered. Finally, several technical details relating to the infinite-dimensional discrete-time positive-real lemma have been relegated to the appendix.

Notation. We define

$$
\mathbb{R}_{+}:=\{x \in \mathbb{R} \mid x \geq 0\}, \quad \mathbb{Z}_{+}=\{x \in \mathbb{Z} \mid x \geq 0\} .
$$

For sets $M$ and $N$, we denote the set of all functions $f: M \rightarrow N$ by $F(M, N)$. If $I \subset \mathbb{R}$ is a compact interval, then $A C(I, \mathbb{R})$ denotes the space of absolutely continuous realvalued functions defined on $I ; A C\left(\mathbb{R}_{+}, \mathbb{R}\right)$ denotes the space of real-valued functions defined on $\mathbb{R}_{+}$which are absolutely continuous on any compact interval $I \subset \mathbb{R}_{+}$, i.e., a function $f \in F\left(\mathbb{R}_{+}, \mathbb{R}\right)$ is in $A C\left(\mathbb{R}_{+}, \mathbb{R}\right)$ if and only if there exists a function $g \in L_{\text {loc }}^{1}\left(\mathbb{R}_{+}, \mathbb{R}\right)$ such that

$$
f(t)=f(0)+\int_{0}^{t} g(\tau) d \tau \quad \forall t \geq 0 .
$$

We say that a function $f \in F\left(\mathbb{R}_{+}, \mathbb{R}\right)$ is piecewise monotone if there exists a sequence $0=t_{0}<t_{1}<t_{2}<\cdots$ such that $\lim _{i \rightarrow \infty} t_{i}=\infty$ and $f$ is monotone on each of the intervals $\left(t_{i}, t_{i+1}\right)$. A function $f \in F\left(\mathbb{R}_{+}, \mathbb{R}\right)$ is called piecewise continuous if there exists a sequence $0=t_{0}<t_{1}<t_{2}<\cdots$ such that $\lim _{i \rightarrow \infty} t_{i}=\infty, f$ is continuous on each of the intervals $\left(t_{i}, t_{i+1}\right)$, and the right and left limits of $f$ exist and are finite at each $t_{i}$. We denote the space of all piecewise continuous functions $f: \mathbb{R}_{+} \rightarrow \mathbb{R}$ by $P C\left(\mathbb{R}_{+}, \mathbb{R}\right)$. As usual, for $f \in P C\left(\mathbb{R}_{+}, \mathbb{R}\right)$, we define

$$
f(t+):=\lim _{\tau \downarrow t} f(\tau)(\text { for } t \geq 0) \quad \text { and } \quad f(t-):=\lim _{\tau \uparrow t} f(\tau)(\text { for } t>0) .
$$

Let $\mathbb{T}=\mathbb{R}_{+}, \mathbb{Z}_{+}$; a function $f \in F(\mathbb{T}, \mathbb{R})$ is called ultimately constant if there exists $T \in \mathbb{T}$ such that $f$ is constant on $[T, \infty) \cap \mathbb{T}$. $L(X, Y)$ denotes the space of bounded linear operators from a Banach space $X$ to a Banach space $Y$, and we set $L(X):=$ $L(X, X)$. The Laplace transform is denoted by $\mathfrak{L}$.

2. A class of discrete-time nonlinear operators. For each $n \in \mathbb{Z}_{+}$, we define a projection operator $\mathbf{Q}_{n}: F\left(\mathbb{Z}_{+}, \mathbb{R}\right) \rightarrow F\left(\mathbb{Z}_{+}, \mathbb{R}\right)$ by

$$
\left(\mathbf{Q}_{n} u\right)(m)= \begin{cases}u(m) & \text { for } m \in[0, n] \cap \mathbb{Z}_{+} \\ u(n) & \text { for } m \in \mathbb{Z}_{+} \backslash[0, n]\end{cases}
$$


Recall that an operator $\Phi: F\left(\mathbb{Z}_{+}, \mathbb{R}\right) \rightarrow F\left(\mathbb{Z}_{+}, \mathbb{R}\right)$ is called causal if for all $u, v \in$ $F\left(\mathbb{Z}_{+}, \mathbb{R}\right)$ and all $n \in \mathbb{Z}_{+}$with $u(m)=v(m)$ for all $m \in[0, n] \cap \mathbb{Z}_{+}$it follows that $(\Phi(u))(m)=(\Phi(v))(m)$ for all $m \in[0, n] \cap \mathbb{Z}_{+}$.

Let $u \in F\left(\mathbb{Z}_{+}, \mathbb{R}\right)$. The function $u$ is called ultimately nondecreasing if there exists $m \in \mathbb{Z}_{+}$such that $u$ is nondecreasing on $\mathbb{Z}_{+} \backslash[0, m]$.

The numerical value set $\operatorname{NVS} \Phi$ of an operator $\Phi: F\left(\mathbb{Z}_{+}, \mathbb{R}\right) \rightarrow F\left(\mathbb{Z}_{+}, \mathbb{R}\right)$ is defined by

$$
\operatorname{NVS} \Phi:=\left\{(\Phi(u))(n) \mid u \in F\left(\mathbb{Z}_{+}, \mathbb{R}\right), n \in \mathbb{Z}_{+}\right\} .
$$

We introduce the following five assumptions on the operator $\Phi: F\left(\mathbb{Z}_{+}, \mathbb{R}\right) \rightarrow$ $F\left(\mathbb{Z}_{+}, \mathbb{R}\right)$ :

(D1) $\Phi$ is causal;

(D2) for all $u \in F\left(\mathbb{Z}_{+}, \mathbb{R}\right)$ and all $n \in \mathbb{Z}_{+}$

$$
\left(\Phi\left(\mathbf{Q}_{n} u\right)\right)(k)=\left(\Phi\left(\mathbf{Q}_{n} u\right)\right)(n) \quad \forall k \in \mathbb{Z}_{+} \backslash[0, n] ;
$$

(D3) there exists $\lambda>0$ such that for all $u \in F\left(\mathbb{Z}_{+}, \mathbb{R}\right)$ and all $n \in \mathbb{Z}_{+}$

$$
u(n) \neq u(n+1) \Longrightarrow \frac{(\Phi(u))(n+1)-(\Phi(u))(n)}{u(n+1)-u(n)} \in[0, \lambda]
$$

(D4) if $u \in F\left(\mathbb{Z}_{+}, \mathbb{R}\right)$ is ultimately nondecreasing and $\lim _{n \rightarrow \infty} u(n)=\infty$, then $(\Phi(u))(n)$ and $(\Phi(-u))(n)$ converge to sup NVS $\Phi$ and inf NVS $\Phi$, respectively, as $n \rightarrow \infty$;

(D5) if, for $u \in F\left(\mathbb{Z}_{+}, \mathbb{R}\right), L:=\lim _{n \rightarrow \infty}(\Phi(u))(n)$ exists with $L \in \operatorname{int}(\operatorname{clos}(\operatorname{NVS} \Phi))$, then $u$ is bounded.

REMARK 2.1. (1) We note that if (D1) and (D2) hold, then (D3) is implied by the monotonicity condition

$$
[(\Phi(u))(n+1)-(\Phi(u))(n)][u(n+1)-u(n)] \geq 0 \quad \forall u \in F\left(\mathbb{Z}_{+}, \mathbb{R}\right), \quad \forall n \in \mathbb{Z}_{+},
$$

together with the Lipschitz continuity condition

$$
\sup _{n \in \mathbb{Z}_{+}}|(\Phi(u))(n)-(\Phi(v))(n)| \leq \lambda \sup _{n \in \mathbb{Z}_{+}}|u(n)-v(n)| \quad \forall u, v \in F\left(\mathbb{Z}_{+}, \mathbb{R}\right) .
$$

(2) Assumption (D2) says that if the input $u$ of the nonlinearity $\Phi$ is constant on $\mathbb{Z}_{+} \backslash[0, n-1]$, then the output $\Phi(u)$ is constant and equal to $(\Phi(u))(n)$ on $\mathbb{Z}_{+} \backslash[0, n-1]$.

(3) If (D1)-(D3) hold, then

$$
|(\Phi(u))(n+1)-(\Phi(u))(n)| \leq \lambda|u(n+1)-u(n)| \quad \forall u \in F\left(\mathbb{Z}_{+}, \mathbb{R}\right), \quad \forall n \in \mathbb{Z}_{+} .
$$

Thus if (D4) also holds, clos (NVS $\Phi)$ is an interval. However, it can be shown that NVS $\Phi$ is not necessarily an interval; see Mawby [21] for a counterexample.

(4) If (D1)-(D3) hold, then for all $u \in F\left(\mathbb{Z}_{+}, \mathbb{R}\right)$ there exists $d: \mathbb{Z}_{+} \rightarrow[0, \lambda]$ such that $(\Phi(u))(n+1)-(\Phi(u))(n)=d(n)(u(n+1)-u(n))$ for all $n \in \mathbb{Z}_{+}$.

If $\Phi: F\left(\mathbb{Z}_{+}, \mathbb{R}\right) \rightarrow F\left(\mathbb{Z}_{+}, \mathbb{R}\right)$ satisfies (D3), then any number $l>0$ such that (D3) holds for $\lambda=l$ is called a Lipschitz constant of $\Phi$. We are now in a position to define the class of nonlinear operators we will be considering in the context of the discrete-time integral control problem in section 3.

Definition 2.2. Let $\lambda>0$. The set of all operators $\Phi: F\left(\mathbb{Z}_{+}, \mathbb{R}\right) \rightarrow F\left(\mathbb{Z}_{+}, \mathbb{R}\right)$ satisfying (D1)-(D5) and having Lipschitz constant $\lambda$ is denoted by $\mathcal{D}(\lambda)$. 


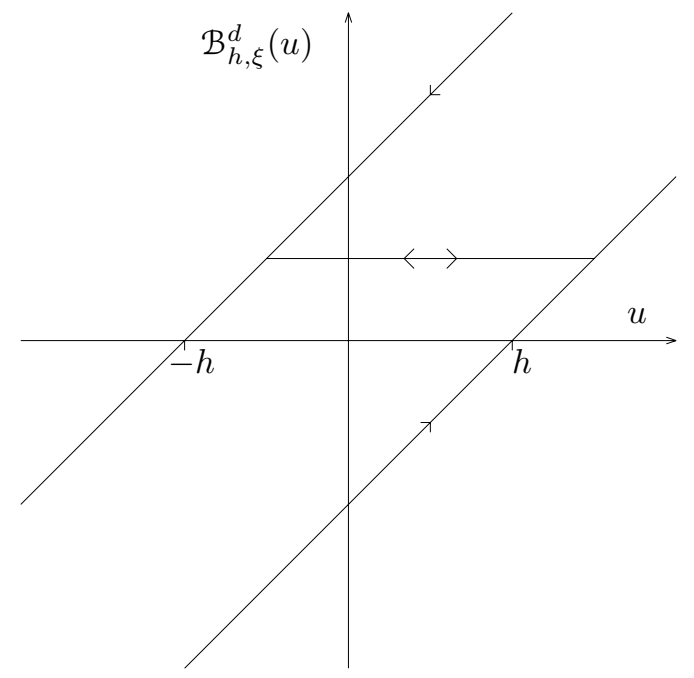

FIG. 3. Backlash hysteresis.

We now consider two examples of nonlinearities which satisfy (D1)-(D5).

Static nonlinearities. For a function $f: \mathbb{R} \rightarrow \mathbb{R}$, define the corresponding static nonlinearity by

$$
\mathcal{S}_{f}: F\left(\mathbb{Z}_{+}, \mathbb{R}\right) \rightarrow F\left(\mathbb{Z}_{+}, \mathbb{R}\right), \quad u \mapsto f \circ u .
$$

It is clear that if $f: \mathbb{R} \rightarrow \mathbb{R}$ is nondecreasing and globally Lipschitz with Lipschitz constant $\lambda>0$, then $\mathcal{S}_{f} \in \mathcal{D}(\lambda)$.

Backlash hysteresis. Let $h \in \mathbb{R}_{+}$be arbitrary. Define the function $b_{h}: \mathbb{R}^{2} \rightarrow \mathbb{R}$ by

$$
b_{h}(v, w)=\max \{v-h, \min \{v+h, w\}\}
$$

We note that

$$
\begin{gathered}
b_{h}(v, w) \in[v-h, v+h] \quad \forall v, w \in \mathbb{R}, \\
b_{h}(v, w)=w \quad \forall(v, w) \in\left\{\left(z_{1}, z_{2}\right) \mid z_{1} \in \mathbb{R}, z_{2} \in\left[z_{1}-h, z_{1}+h\right]\right\}, \\
\left(b_{h}\left(v_{1}, w\right)-b_{h}\left(v_{2}, w\right)\right)\left(v_{1}-v_{2}\right) \geq 0 \quad \forall v_{1}, v_{2}, w \in \mathbb{R} .
\end{gathered}
$$

For each $\xi \in \mathbb{R}$, we introduce the discrete-time backlash operator $\mathcal{B}_{h, \xi}^{d}: F\left(\mathbb{Z}_{+}, \mathbb{R}\right) \rightarrow$ $F\left(\mathbb{Z}_{+}, \mathbb{R}\right)$ by defining recursively

$$
\left(\mathcal{B}_{h, \xi}^{d}(u)\right)(n)= \begin{cases}b_{h}(u(0), \xi) & \text { for } n=0 \\ b_{h}\left(u(n),\left(\mathcal{B}_{h, \xi}^{d}(u)\right)(n-1)\right) & \text { for } n \in \mathbb{Z}_{+} \backslash\{0\} .\end{cases}
$$

We remark that $\xi$ plays the role of an "initial state." The discrete-time backlash operator $\mathcal{B}_{h, \xi}^{d}$ is illustrated in Figure 3 . 
We show that $\mathcal{B}_{h, \xi}^{d} \in \mathcal{D}(1)$. It is immediately clear from the definition that $\mathcal{B}_{h, \xi}^{d}$ satisfies (D1). Using (2.2) and (2.3), we see that (D2) holds. Combining (2.1)-(2.4) leads to

$\left[\left(\mathcal{B}_{h, \xi}^{d}(u)\right)(n+1)-\left(\mathcal{B}_{h, \xi}^{d}(u)\right)(n)\right][u(n+1)-u(n)] \geq 0 \quad \forall u \in F\left(\mathbb{Z}_{+}, \mathbb{R}\right), \quad \forall n \in \mathbb{Z}_{+}$.

It is not difficult to show (see $[2$, p. 42$]$ ) that

$$
\left|b_{h}\left(v_{1}, w_{1}\right)-b_{h}\left(v_{2}, w_{2}\right)\right| \leq \max \left(\left|v_{1}-v_{2}\right|,\left|w_{1}-w_{2}\right|\right) \quad \forall v_{1}, v_{2}, w_{1}, w_{2} \in \mathbb{R} .
$$

Thus

$$
\left|\left(\mathcal{B}_{h, \xi}^{d}(u)\right)(n+1)-\left(\mathcal{B}_{h, \xi}^{d}(u)\right)(n)\right| \leq|u(n+1)-u(n)| \quad \forall u \in F\left(\mathbb{Z}_{+}, \mathbb{R}\right), \quad \forall n \in \mathbb{Z}_{+},
$$

which, combined with (2.5), implies that (D3) holds for $\lambda=1$. Note that NVS $\mathcal{B}_{h, \xi}^{d}=$ $\mathbb{R}$. By $(2.2)$, for all $u \in F\left(\mathbb{Z}_{+}, \mathbb{R}\right)$ and all $n \in \mathbb{Z}_{+},\left(\mathcal{B}_{h, \xi}^{d}(u)\right)(n) \in[u(n)-h, u(n)+h]$, showing that (D4) holds. Finally, it is clear that

$$
v \in\left[b_{h}(v, w)-h, b_{h}(v, w)+h\right] \quad \forall v, w \in \mathbb{R},
$$

and so

$$
u(n) \in\left[\left(\mathcal{B}_{h, \xi}^{d}(u)\right)(n)-h,\left(\mathcal{B}_{h, \xi}^{d}(u)\right)(n)+h\right] \quad \forall u \in F\left(\mathbb{Z}_{+}, \mathbb{R}\right), \quad \forall n \in \mathbb{Z}_{+},
$$

showing that (D5) is satisfied. We have shown that (D1)-(D5) hold for $\mathcal{B}_{h, \xi}^{d}$ (with $\lambda=1$ ), and hence $\mathcal{B}_{h, \xi}^{d} \in \mathcal{D}(1)$. We direct the reader to section 4 for a discussion of the backlash operator in a continuous-time setting.

3. Discrete-time integral control. Consider a single-input, single-output, discrete-time system

$$
\begin{aligned}
x(n+1) & =A x(n)+B u(n), \quad x(0)=x_{0} \in X, \\
y(n) & =C x(n)+D u(n),
\end{aligned}
$$

evolving on a real Hilbert space $X$. Here $A \in L(X), B \in L(\mathbb{R}, X), C \in L(X, \mathbb{R})$, and $D \in \mathbb{R}$. A system of the form (3.1) is called power-stable if $A$ is power-stable, i.e., there exist $M \geq 1$ and $\theta \in(0,1)$ such that

$$
\left\|A^{n}\right\| \leq M \theta^{n} \quad \forall n \in \mathbb{Z}_{+} .
$$

The transfer function $\mathbf{G}$ of (3.1) is given by

$$
\mathbf{G}(z)=C(z I-A)^{-1} B+D .
$$

Suppose that system (3.1) is subject to a causal input nonlinearity $\Phi: F\left(\mathbb{Z}_{+}, \mathbb{R}\right) \rightarrow$ $F\left(\mathbb{Z}_{+}, \mathbb{R}\right)$, yielding the nonlinear system

$$
\begin{aligned}
x(n+1) & =A x(n)+B(\Phi(u))(n), \quad x(0)=x_{0} \in X, \\
y(n) & =C x(n)+D(\Phi(u))(n) .
\end{aligned}
$$

Denoting the reference value by $r$, the control law

$$
u(n+1)=u(n)+k(r-y(n)),
$$


where $k$ is a real parameter, then leads to the following nonlinear system of difference equations:

$$
\begin{aligned}
& x(n+1)=A x(n)+B(\Phi(u))(n), \quad x(0)=x_{0} \in X, \\
& u(n+1)=u(n)+k(r-C x(n)-D(\Phi(u))(n)), \quad u(0)=u_{0} \in \mathbb{R} .
\end{aligned}
$$

If $\mathbf{G}$ is holomorphic and bounded on $\{z \in \mathbb{C}|| z \mid>\alpha\}$ for some $\alpha<1$ (which is the case if (3.1) is power-stable) and $\mathbf{G}(1)>0$, then it can be shown that

$$
1+k \operatorname{Re} \frac{\mathbf{G}(z)}{z-1} \geq 0 \quad \forall|z|>1,
$$

for all sufficiently small $k>0$; see [16]. We define

$$
K:=\sup \{k>0 \mid(3.4) \text { holds }\} .
$$

We can now state the main result of this section.

Theorem 3.1. Let $\lambda>0$. Assume that $\Phi \in \mathcal{D}(\lambda)$, (3.1) is power-stable, $\mathbf{G}(1)>$ $0, k \in(0, K / \lambda)$, and $r \in \mathbb{R}$ is such that $\tilde{r}:=r / \mathbf{G}(1) \in \operatorname{clos}(\mathrm{NVS} \Phi)$. Then for all $\left(x_{0}, u_{0}\right) \in X \times \mathbb{R}$, the solution $(x, u)$ of (3.3) satisfies the following:

(1) $\lim _{n \rightarrow \infty}(\Phi(u))(n)=\tilde{r}$;

(2) $\lim _{n \rightarrow \infty} x(n)=(I-A)^{-1} B \tilde{r}$;

(3) $\lim _{n \rightarrow \infty} y(n)=r$, where $y(n)=C x(n)+D(\Phi(u))(n)$;

(4) if $\tilde{r} \in \operatorname{int}(\operatorname{clos}(\operatorname{NVS} \Phi))$, then $u$ is bounded.

Proof. Denote the solution of (3.3) by $(x, u)$, and introduce new variables by defining

$$
z(n):=x(n)-(I-A)^{-1} B(\Phi(u))(n), \quad v(n):=(\Phi(u))(n)-\tilde{r} \quad \forall n \in \mathbb{Z}_{+} .
$$

By Remark 2.1, part (4), there exists $d: \mathbb{Z}_{+} \rightarrow[0, \lambda]$ such that $(\Phi(u))(n+1)-$ $(\Phi(u))(n)=d(n)(u(n+1)-u(n))$ for all $n \in \mathbb{Z}_{+}$. Using the identity $A(I-A)^{-1}=$ $(I-A)^{-1}-I$, a straightforward calculation yields

$$
\begin{aligned}
& z(n+1)=A z(n)-(I-A)^{-1} B w(n), \quad z(0)=z_{0}, \\
& v(n+1)=v(n)+w(n), \quad v(0)=v_{0},
\end{aligned}
$$

where

$$
w(n)=-k d(n)(C z(n)+\mathbf{G}(1) v(n))
$$

and

$$
z_{0}:=x_{0}-(I-A)^{-1} B(\Phi(u))(0), \quad v_{0}:=(\Phi(u))(0)-\tilde{r} .
$$

Choose $c \in(k \lambda, K)$, and define

$$
\mathbf{H}(z)=-C(z I-A)^{-1}(I-A)^{-1} B+J,
$$

where $J:=1 / c-\mathbf{G}(1) / 2$. Then

$$
\mathbf{H}(z)=\frac{1}{z-1}(\mathbf{G}(z)-\mathbf{G}(1))+J .
$$


Since $c<K$, there exists $\varepsilon>0$ such that

$$
\frac{1}{c}+\operatorname{Re} \frac{\mathbf{G}(z)}{z-1} \geq \varepsilon \quad \forall|z|>1,
$$

and hence, using the identity

$$
\operatorname{Re}\left(\frac{1}{e^{i \theta}-1}\right)=-\frac{1}{2} \quad \forall \theta \in(0,2 \pi)
$$

we may conclude that

$$
\operatorname{Re} \mathbf{H}\left(e^{i \theta}\right) \geq \varepsilon \quad \forall \theta \in[0,2 \pi) .
$$

An application of the discrete-time positive-real lemma (see the appendix) shows that there exist $P \in L(X), P=P^{*} \geq 0, L \in L(\mathbb{R}, X)$, and $W \in \mathbb{R}$ such that

$$
\begin{aligned}
A^{*} P A-P & =-L L^{*}, \\
A^{*} P(I-A)^{-1} B & =L W-C^{*} \\
W^{2} & =2 J-B^{*}\left(I-A^{*}\right)^{-1} P(I-A)^{-1} B .
\end{aligned}
$$

For $n \in \mathbb{Z}_{+}$, define

$$
V(n)=\langle z(n), P z(n)\rangle+\mathbf{G}(1) v(n)^{2} .
$$

Using (3.6) and (3.7), we obtain for all $n \in \mathbb{Z}_{+}$

$$
\begin{aligned}
V(n+1)-V(n)=\langle z(n+1), P z(n+1)\rangle-\langle z(n), P z(n)\rangle+\mathbf{G}(1)\left(v(n+1)^{2}-v(n)^{2}\right) \\
=-\left(L^{*} z(n)\right)^{2}-2\left(L^{*} z(n)\right) W w(n)+2(C z(n)) w(n) \\
\quad \quad+w(n)\left(2 J-W^{2}\right) w(n)+\mathbf{G}(1)\left(w(n)^{2}+2 w(n) v(n)\right) \\
=-\left(L^{*} z(n)\right)^{2}-(W w(n))^{2}-2\left(L^{*} z(n)\right) W w(n) \\
\quad \quad+2(C z(n)) w(n)+\frac{2}{c} w(n)^{2}+2 \mathbf{G}(1) w(n) v(n) \\
=-\left(L^{*} z(n)+W w(n)\right)^{2}+2(C z(n)) w(n) \\
\quad \quad+\frac{2}{c} w(n)^{2}-2 \mathbf{G}(1) k d(n)\left[\mathbf{G}(1) v(n)^{2}+(C z(n)) v(n)\right] \\
=-\left(L^{*} z(n)+W w(n)\right)^{2}+\frac{2}{c} w(n)^{2}-2 k d(n)(\mathbf{G}(1) v(n)+C z(n))^{2} \\
=-\left(L^{*} z(n)+W w(n)\right)^{2}-2\left(k d(n)-\frac{k^{2} d(n)^{2}}{c}\right)(\mathbf{G}(1) v(n)+C z(n))^{2} .
\end{aligned}
$$

Summing from $n=0$ to $n=\infty$ then gives

$$
2 \sum_{n=0}^{\infty}\left(k d(n)-\frac{k^{2} d(n)^{2}}{c}\right)(\mathbf{G}(1) v(n)+C z(n))^{2} \leq V(0)<\infty .
$$

Now, since $c>k \lambda$ and $d(n) \in[0, \lambda]$, we have

$$
k d(n)-\frac{k^{2} d(n)^{2}}{c}=k d(n)\left(1-\frac{k d(n)}{c}\right) \geq k d(n)\left(1-\frac{k \lambda}{c}\right) \geq k \frac{\delta}{\lambda} d(n)^{2} \quad \forall n \in \mathbb{Z}_{+},
$$


where $\delta:=1-k \lambda / c>0$. Therefore, (3.8) implies that

$$
d(C z+\mathbf{G}(1) v) \in l^{2}\left(\mathbb{Z}_{+}\right),
$$

and hence

$$
w \in l^{2}\left(\mathbb{Z}_{+}\right) .
$$

Appealing to the fact that $A$ is power-stable, we may conclude from (3.6a) and (3.10) that

$$
z \in l^{2}\left(\mathbb{Z}_{+}\right)
$$

Consequently, $C z \in l^{2}\left(\mathbb{Z}_{+}\right)$, and hence, by (3.9) and the boundedness of $d$,

$$
d v \in l^{2}\left(\mathbb{Z}_{+}\right)
$$

From (3.11) and (3.12) we obtain that

$$
(C z) d v \in l^{1}\left(\mathbb{Z}_{+}\right) .
$$

Using (3.8), (3.11)-(3.13), and the boundedness of $d$ it follows that

$$
d v^{2} \in l^{1}\left(\mathbb{Z}_{+}\right) .
$$

It follows from (3.6b) that, for all $m \in \mathbb{Z}_{+}$,

$$
v(m+1)^{2}=v(0)^{2}+\sum_{n=0}^{m} w(n)^{2}+2 \sum_{n=0}^{m} v(n) w(n) .
$$

Combining (3.15) with (3.10), (3.13), and (3.14) and recalling that $w=-k d(C z+$ $\mathbf{G}(1) v)$, we see that there exists a number $\nu \in \mathbb{R}_{+}$such that

$$
\lim _{n \rightarrow \infty} v(n)^{2}=\nu
$$

In order to prove statement (1), it is sufficient to show that $\nu=0$. Seeking a contradiction, suppose that $\nu>0$. By $(3.10), \lim _{n \rightarrow \infty} w(n)=0$, and thus we may conclude from (3.6b) that

$$
\lim _{n \rightarrow \infty}(v(n+1)-v(n))=0 .
$$

Since $\nu>0,(3.16)$ and (3.17) yield that $v(n)$ does not change sign for sufficiently large $n$, and so

$$
\lim _{n \rightarrow \infty} v(n)=\sqrt{\nu} \quad \text { or } \quad \lim _{n \rightarrow \infty} v(n)=-\sqrt{\nu} .
$$

Assuming that $\lim _{n \rightarrow \infty} v(n)=-\sqrt{\nu}$ (the case $\lim _{n \rightarrow \infty} v(n)=\sqrt{\nu}$ can be dealt with in an entirely analogous fashion), we obtain that

$$
\Phi_{\infty}:=\lim _{n \rightarrow \infty}(\Phi(u))(n)<\tilde{r},
$$

and thus

$$
\lim _{n \rightarrow \infty} x(n)=(I-A)^{-1} B \Phi_{\infty} .
$$


It then follows from (3.3b), (3.18), and (3.19) that

$$
\lim _{n \rightarrow \infty}(u(n+1)-u(n))=k\left(r-C(I-A)^{-1} B \Phi_{\infty}-D \Phi_{\infty}\right)=k \mathbf{G}(1)\left(\tilde{r}-\Phi_{\infty}\right)>0 .
$$

Therefore, $\lim _{n \rightarrow \infty} u(n)=\infty$, and $u$ is ultimately nondecreasing, so by (D4) and the assumption that $\tilde{r} \in \operatorname{clos}(\operatorname{NVS} \Phi)$ we obtain

$$
\Phi_{\infty}=\lim _{n \rightarrow \infty}(\Phi(u))(n)=\sup (\operatorname{NVS} \Phi) \geq \tilde{r}
$$

contradicting (3.18). Therefore, $\lim _{n \rightarrow \infty} v(n)=0$, and, consequently, $\lim _{n \rightarrow \infty}(\Phi(u))(n)$ $=\tilde{r}$, which is statement $(1)$.

Statement (2) follows immediately from statement (1). Statement (3) is an easy consequence of statements (1) and (2). Finally, to prove statement (4), let $\tilde{r} \in \operatorname{int}(\operatorname{clos}(\operatorname{NVS} \Phi))$. Then the boundedness of $u$ follows immediately from statement (1) and (D5).

We see from the proof of Theorem 3.1 that (D5) is needed only for statement (4).

One of the conditions imposed in Theorem 3.1 is that $r / \mathbf{G}(1) \in \operatorname{clos}(\operatorname{NVS} \Phi)$. The following proposition shows that this condition is close to being necessary for tracking insofar as, if tracking of $r$ is achievable while maintaining the boundedness of $\Phi(u)$, then $r / \mathbf{G}(1) \in \operatorname{clos}(\operatorname{NVS} \Phi)$.

Proposition 3.2. Let $\lambda>0$ and $r \in \mathbb{R}$. Suppose that $\Phi \in \mathcal{D}(\lambda)$, $A$ is powerstable, and $\mathbf{G}(1)>0$. If there exist an initial condition $x_{0} \in X$ and a function $u \in F\left(\mathbb{Z}_{+}, \mathbb{R}\right)$ such that $\Phi(u)$ is bounded and

$$
\lim _{n \rightarrow \infty}[C x(n)+D(\Phi(u))(n)]=r,
$$

where $x \in F\left(\mathbb{Z}_{+}, X\right)$ is given by $(3.2 \mathrm{a})$, then $r / \mathbf{G}(1) \in \operatorname{clos}(\operatorname{NVS} \Phi)$.

Proof. Since $\Phi(u)$ is bounded and $A$ is power-stable, $x$ is bounded. Let $n \in \mathbb{Z}_{+}$, and define $y: \mathbb{Z}_{+} \rightarrow \mathbb{R}$ by $(3.2 \mathrm{~b})$; then

$$
y(n)=C\left(x(n)-(I-A)^{-1} B(\Phi(u))(n)\right)+\mathbf{G}(1)(\Phi(u))(n),
$$

and therefore

$$
C(A-I)^{-1}(x(n+1)-x(n))=y(n)-\mathbf{G}(1)(\Phi(u))(n) .
$$

For $p, m \in \mathbb{Z}_{+}$with $p>m$, summing the above from $m$ to $p-1$ gives

$$
C(A-I)^{-1}(x(p)-x(m))=\sum_{k=m}^{p-1}(y(k)-\mathbf{G}(1)(\Phi(u))(k)) .
$$

Seeking a contradiction, let us suppose that $r / \mathbf{G}(1) \notin \operatorname{clos}(\operatorname{NVS} \Phi)$. Since $\lim _{n \rightarrow \infty} y(n)$ $=r$ and $\operatorname{clos}(\operatorname{NVS} \Phi)$ is an interval (see Remark 2.1, part (3)), there exist $\varepsilon>0$, $\beta \in\{-1,1\}$, and $m \in \mathbb{Z}_{+}$such that

$$
\beta(y(n)-\mathbf{G}(1)(\Phi(u))(n)) \geq \varepsilon \quad \forall n \geq m .
$$

Combining the above with (3.21), it follows that

$$
\beta C(A-I)^{-1}(x(n)-x(m))=\sum_{k=m}^{n-1} \beta(y(k)-\mathbf{G}(1)(\Phi(u))(k)) \geq \varepsilon(n-m) \quad \forall n>m .
$$

Therefore, $\lim _{n \rightarrow \infty} \beta C(A-I)^{-1} x(n)=\infty$, contradicting the boundedness of $x$. 
4. A class of continuous-time hysteresis operators and their discretizations. We call a function $f: \mathbb{R}_{+} \rightarrow \mathbb{R}_{+}$a time transformation if $f$ is continuous and nondecreasing and satisfies $f(0)=0$ and $\lim _{t \rightarrow \infty} f(t)=\infty$, i.e., $f$ is continuous, nondecreasing, and surjective. We denote the set of all time transformations $f: \mathbb{R}_{+} \rightarrow \mathbb{R}_{+}$by $\mathcal{T}$. For all $\tau \in \mathbb{R}_{+}$, we define a (continuous-time) projection operator $\mathbf{Q}_{\tau}^{c}: F\left(\mathbb{R}_{+}, \mathbb{R}\right) \rightarrow F\left(\mathbb{R}_{+}, \mathbb{R}\right)$ by

$$
\left(\mathbf{Q}_{\tau}^{c} v\right)(t)= \begin{cases}v(t) & \text { for } 0 \leq t \leq \tau \\ v(\tau) & \text { for } t>\tau .\end{cases}
$$

In the following, let $\mathcal{F} \subset F\left(\mathbb{R}_{+}, \mathbb{R}\right), \mathcal{F} \neq \emptyset$. We introduce the following two assumptions on $\mathcal{F}$ :

(F1) $v \circ f \in \mathcal{F}$ for all $v \in \mathcal{F}$ and all $f \in \mathcal{T}$;

(F2) $\mathbf{Q}_{t}^{c}(\mathcal{F}) \subset \mathcal{F}$ for all $t \in \mathbb{R}_{+}$.

We call an operator $\Phi: \mathcal{F} \rightarrow F\left(\mathbb{R}_{+}, \mathbb{R}\right)$ causal if for all $v, w \in \mathcal{F}$ and all $\tau \in \mathbb{R}_{+}$ with $v(t)=w(t)$ for all $t \in[0, \tau]$ it follows that $(\Phi(v))(t)=(\Phi(w))(t)$ for all $t \in[0, \tau]$. An operator $\Phi: \mathcal{F} \rightarrow F\left(\mathbb{R}_{+}, \mathbb{R}\right)$ is called rate independent if $\mathcal{F}$ satisfies $(\mathrm{F} 1)$ and

$$
(\Phi(v \circ f))(t)=(\Phi(v))(f(t)) \quad \forall v \in \mathcal{F}, \quad \forall f \in \mathcal{T}, \quad \forall t \in \mathbb{R}_{+} .
$$

A functional $\varphi: \mathcal{F} \rightarrow \mathbb{R}$ is called rate independent if $\mathcal{F}$ satisfies $(\mathrm{F} 1)$ and

$$
\varphi(u \circ f)=\varphi(u) \quad \forall u \in \mathcal{F}, \quad \forall f \in \mathcal{T} .
$$

Definition 4.1. Let $\mathcal{F} \subset F\left(\mathbb{R}_{+}, \mathbb{R}\right), \mathcal{F} \neq \emptyset$. An operator $\Phi: \mathcal{F} \rightarrow F\left(\mathbb{R}_{+}, \mathbb{R}\right)$ is called a hysteresis operator if $\mathcal{F}$ satisfies $(\mathrm{F} 1)$ and $\Phi$ is causal and rate independent.

For $\mathcal{F} \subset F\left(\mathbb{R}_{+}, \mathbb{R}\right), \mathcal{F} \neq \emptyset$, let $\mathcal{F}^{\text {uc }}$ denote the set of all ultimately constant $u \in \mathcal{F}$, i.e.,

$$
\mathcal{F}^{\mathrm{uc}}=\{u \in \mathcal{F} \mid u \text { is ultimately constant }\} .
$$

Clearly, if $\mathcal{F}$ satisfies (F2), then $\mathcal{F}^{\mathrm{uc}} \neq \emptyset$. Moreover, if $\mathcal{F}$ satisfies (F1), then so does $\mathcal{F}^{\mathrm{uc}}$. The proof of the following proposition can be found in [10].

Proposition 4.2. Let $\mathcal{F} \subset F\left(\mathbb{R}_{+}, \mathbb{R}\right), \mathcal{F} \neq \emptyset$, and assume that $(\mathrm{F} 1)$ and $(\mathrm{F} 2)$ are satisfied. If $\Phi: \mathcal{F} \rightarrow F\left(\mathbb{R}_{+}, \mathbb{R}\right)$ is a hysteresis operator, then the following statements hold:

(1) for all $v \in \mathcal{F}$ and all $\tau \in \mathbb{R}_{+}$

$$
\left(\Phi\left(\mathbf{Q}_{\tau}^{c} v\right)\right)(t)=(\Phi(v))(\tau) \quad \forall t \geq \tau ;
$$

(2) the functional

$$
\varphi: \mathcal{F}^{\mathrm{uc}} \rightarrow \mathbb{R}, \quad v \mapsto \lim _{t \rightarrow \infty}(\Phi(v))(t)
$$

is rate independent and satisfies

$$
(\Phi(v))(t)=\varphi\left(\mathbf{Q}_{t}^{c} v\right) \quad \forall v \in \mathcal{F}, \quad \forall t \in \mathbb{R}_{+} .
$$

Conversely, if $\varphi: \mathcal{F}^{\mathrm{uc}} \rightarrow \mathbb{R}$ is a rate independent functional, then $\Phi: \mathcal{F} \rightarrow F\left(\mathbb{R}_{+}, \mathbb{R}\right)$ given by (4.2) is a hysteresis operator and satisfies

$$
\lim _{t \rightarrow \infty}(\Phi(v))(t)=\varphi(v) \quad \forall v \in \mathcal{F}^{\mathrm{uc}} .
$$


For a hysteresis operator $\Phi: \mathcal{F} \rightarrow F\left(\mathbb{R}_{+}, \mathbb{R}\right)$, we call the rate independent functional $\varphi$ defined by (4.1) the representing functional of $\Phi$.

For any $v \in F\left(\mathbb{R}_{+}, \mathbb{R}\right)$ and any $t \in \mathbb{R}_{+}$, we define

$$
M(v, t):=\{\tau \in(t, \infty) \mid v \text { is monotone on }(t, \tau)\} .
$$

If $v$ is piecewise monotone, then $M(v, t) \neq \emptyset$ for all $t \in \mathbb{R}_{+}$and the standard monotonicity partition $t_{0}<t_{1}<t_{2}<\cdots$ of $v$ is defined recursively by setting $t_{0}=0$ and $t_{i+1}=\sup M\left(v, t_{i}\right)$ for all $i \in \mathbb{Z}_{+}$such that $M\left(v, t_{i}\right)$ is bounded. If $v$ is piecewise monotone and ultimately constant, then the standard monotonicity partition of $v$ is finite.

The space of all piecewise monotone $v \in C\left(\mathbb{R}_{+}, \mathbb{R}\right)$ is denoted by $C_{\mathrm{pm}}\left(\mathbb{R}_{+}, \mathbb{R}\right)$. We define $C_{\mathrm{pm}}^{\mathrm{uc}}\left(\mathbb{R}_{+}, \mathbb{R}\right)$ to be the space of all ultimately constant $v \in C_{\mathrm{pm}}\left(\mathbb{R}_{+}, \mathbb{R}\right)$. Let $F^{\text {uc }}\left(\mathbb{Z}_{+}, \mathbb{R}\right)$ denote the space of ultimately constant $v: \mathbb{Z}_{+} \rightarrow \mathbb{R}$. We define the restriction operator $R: C_{\mathrm{pm}}^{\mathrm{uc}}\left(\mathbb{R}_{+}, \mathbb{R}\right) \rightarrow F^{\mathrm{uc}}\left(\mathbb{Z}_{+}, \mathbb{R}\right)$ by

$$
(R(v))(k)= \begin{cases}v\left(t_{k}\right) & \text { for } k \in[0, m] \cap \mathbb{Z}_{+}, \\ \lim _{t \rightarrow \infty} v(t) & \text { for } k \in \mathbb{Z}_{+} \backslash[0, m]\end{cases}
$$

where $0=t_{0}<t_{1}<\cdots<t_{m}$ is the standard monotonicity partition of $v$.

In the following, we want to extend hysteresis operators defined on $C_{\mathrm{pm}}\left(\mathbb{R}_{+}, \mathbb{R}\right)$ to spaces of piecewise continuous functions. This requires some preparation. For $\tau>0$, we define the prolongation operator $P_{\tau}: F\left(\mathbb{Z}_{+}, \mathbb{R}\right) \rightarrow C_{\mathrm{pm}}\left(\mathbb{R}_{+}, \mathbb{R}\right)$ by letting $P_{\tau} u$ be the linear interpolate for the values $\left(P_{\tau} u\right)(i \tau)=u(i)$. Let $N P C\left(\mathbb{R}_{+}, \mathbb{R}\right) \subset P C\left(\mathbb{R}_{+}, \mathbb{R}\right)$ denote the space of all normalized piecewise continuous functions $v: \mathbb{R}_{+} \rightarrow \mathbb{R}$; that is, $v$ is piecewise continuous and is right-continuous or left-continuous at each point $t \in \mathbb{R}_{+}$. The space of all piecewise monotone functions $v \in N P C\left(\mathbb{R}_{+}, \mathbb{R}\right)$ is denoted by $N P C_{\mathrm{pm}}\left(\mathbb{R}_{+}, \mathbb{R}\right)$, while $N P C_{\mathrm{pm}}^{\mathrm{uc}}\left(\mathbb{R}_{+}, \mathbb{R}\right)$ denotes the space of ultimately constant $v \in N P C_{\mathrm{pm}}\left(\mathbb{R}_{+}, \mathbb{R}\right)$. We note that $N P C_{\mathrm{pm}}\left(\mathbb{R}_{+}, \mathbb{R}\right)$ and $N P C_{\mathrm{pm}}^{\mathrm{uc}}\left(\mathbb{R}_{+}, \mathbb{R}\right)$ both satisfy $(\mathrm{F} 1)$ and $(\mathrm{F} 2)$. For $v \in N P C_{\mathrm{pm}}^{\mathrm{uc}}\left(\mathbb{R}_{+}, \mathbb{R}\right)$, we define $v(\infty):=\lim _{\tau \rightarrow \infty} v(\tau)$.

Let $v \in N P C_{\mathrm{pm}}^{\mathrm{uc}}\left(\mathbb{R}_{+}, \mathbb{R}\right)$, and let $0=t_{0}<t_{1}<\cdots<t_{m}$ be the standard monotonicity partition of $v$. We define the operator $\rho: N P C_{\mathrm{pm}}^{\mathrm{uc}}\left(\mathbb{R}_{+}, \mathbb{R}\right) \rightarrow F^{\mathrm{uc}}\left(\mathbb{Z}_{+}, \mathbb{R}\right)$ by

$$
\rho(v)=\left(v\left(t_{0}\right), v\left(t_{1}-\right), v\left(t_{1}+\right), v\left(t_{2}-\right), v\left(t_{2}+\right), \ldots, v\left(t_{m}-\right), v\left(t_{m}+\right), v(\infty), v(\infty), \ldots\right) .
$$

For $\tau>0$, define

$$
R_{e}: N P C_{\mathrm{pm}}^{\mathrm{uc}}\left(\mathbb{R}_{+}, \mathbb{R}\right) \rightarrow F^{\mathrm{uc}}\left(\mathbb{Z}_{+}, \mathbb{R}\right), \quad v \mapsto R\left(\left(P_{\tau} \circ \rho\right)(v)\right) .
$$

The operator $R_{e}$ is an extension of $R$, and the definition of $R_{e}$ is independent of $\tau$ (see [10]). The function $v$, shown in Figure 4, is a normalized piecewise continuous function which is piecewise monotone and ultimately constant, so $v \in N P C_{\mathrm{pm}}^{\mathrm{uc}}\left(\mathbb{R}_{+}, \mathbb{R}\right)$. It has standard monotonicity partition $0=t_{0}<t_{1}<t_{2}<t_{3}<t_{4}$,

$$
\rho(v)=\left(v_{0}, v_{7}, v_{6}, v_{4}, v_{4}, v_{5}, v_{3}, v_{7}, v_{2}, v_{1}, v_{1}, v_{1}, \ldots\right),
$$

and

$$
R_{e}(v)=\left(v_{0}, v_{7}, v_{4}, v_{5}, v_{3}, v_{7}, v_{1}, v_{1}, v_{1}, \ldots\right) .
$$

For any rate independent $\varphi: C_{\mathrm{pm}}^{\mathrm{uc}}\left(\mathbb{R}_{+}, \mathbb{R}\right) \rightarrow \mathbb{R}$, we define

$$
\varphi_{e}: N P C_{\mathrm{pm}}^{\mathrm{uc}}\left(\mathbb{R}_{+}, \mathbb{R}\right) \rightarrow \mathbb{R}, \quad v \mapsto \varphi\left(\left(P_{\tau} \circ R_{e}\right)(v)\right),
$$




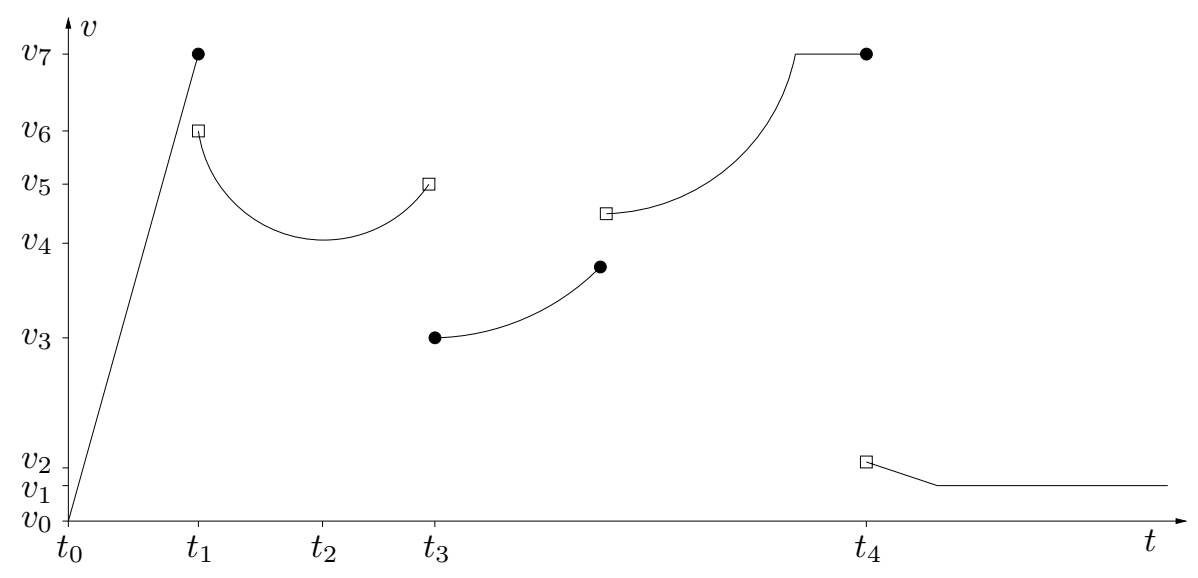

FiG. 4. Example of a function in $N P C_{\mathrm{pm}}^{\mathrm{uc}}\left(\mathbb{R}_{+}, \mathbb{R}\right)$.

where $\tau>0$. From [10] we know that $\varphi_{e}$ does not depend on $\tau$ and that $\varphi_{e}$ is a rate independent extension of $\varphi$.

Let $\Phi: C_{\mathrm{pm}}\left(\mathbb{R}_{+}, \mathbb{R}\right) \rightarrow C\left(\mathbb{R}_{+}, \mathbb{R}\right)$ be a hysteresis operator, and let $\varphi: C_{\mathrm{pm}}^{\mathrm{uc}}\left(\mathbb{R}_{+}, \mathbb{R}\right) \rightarrow$ $\mathbb{R}$ be the representing functional of $\Phi$. Define

$$
\Phi_{e}: N P C_{\mathrm{pm}}\left(\mathbb{R}_{+}, \mathbb{R}\right) \rightarrow F\left(\mathbb{R}_{+}, \mathbb{R}\right)
$$

by setting

$$
\left(\Phi_{e}(v)\right)(t)=\varphi_{e}\left(\mathbf{Q}_{t}^{c} v\right) \quad \forall t \in \mathbb{R}_{+},
$$

where $\varphi_{e}$ is the extension of $\varphi$ to $N P C_{\mathrm{pm}}^{\mathrm{uc}}\left(\mathbb{R}_{+}, \mathbb{R}\right)$ given by (4.4).

Define $\mathcal{S}_{\tau}$ to be the set of all right-continuous step functions $v: \mathbb{R}_{+} \rightarrow \mathbb{R}$ of step length $\tau>0$. The following result was proved in [10].

Proposition 4.3. Let $\Phi: C_{\mathrm{pm}}\left(\mathbb{R}_{+}, \mathbb{R}\right) \rightarrow C\left(\mathbb{R}_{+}, \mathbb{R}\right)$ be a hysteresis operator. Then

(1) $\Phi_{e}$ is an extension of $\Phi$;

(2) $\Phi_{e}$ is a hysteresis operator with representing functional $\varphi_{e}$;

(3) for $v, w \in N P C_{\mathrm{pm}}\left(\mathbb{R}_{+}, \mathbb{R}\right)$ and $t \in \mathbb{R}_{+}$

$$
R_{e}\left(\mathbf{Q}_{t}^{c} v\right)=R_{e}\left(\mathbf{Q}_{t}^{c} w\right) \quad \Longrightarrow \quad\left(\Phi_{e}(v)\right)(t)=\left(\Phi_{e}(w)\right)(t) ;
$$

(4) $\Phi_{e}\left(N P C_{\mathrm{pm}}\left(\mathbb{R}_{+}, \mathbb{R}\right)\right) \subset N P C\left(\mathbb{R}_{+}, \mathbb{R}\right)$;

(5) $\Phi_{e}\left(\mathcal{S}_{\tau}\right) \subset \mathcal{S}_{\tau}$.

For given $v \in \mathcal{S}_{\tau}$ we introduce continuous piecewise monotone "approximations" $v_{k} \in C_{\mathrm{pm}}\left(\mathbb{R}_{+}, \mathbb{R}\right)(k=1,2, \ldots)$ as follows: let $\varepsilon_{k} \in(0, \tau)$ with $\lim _{k \rightarrow \infty} \varepsilon_{k}=0$, and define the following:

(i) if $t \in\left[(i+1) \tau-\varepsilon_{k},(i+1) \tau\right), i \in \mathbb{Z}_{+}$,

$$
v_{k}(t)=v(i \tau)+\frac{v((i+1) \tau)-v(i \tau)}{\varepsilon_{k}}\left(t-(i+1) \tau+\varepsilon_{k}\right) ;
$$

(ii) $v_{k}(t)=v(t)$ otherwise.

(See Figure 5 for an illustration.)

The following result shows that, for a given $v \in \mathcal{S}_{\tau}$, the functions $\Phi\left(v_{k}\right)$ approximate $\Phi_{e}(v)$ pointwise. 


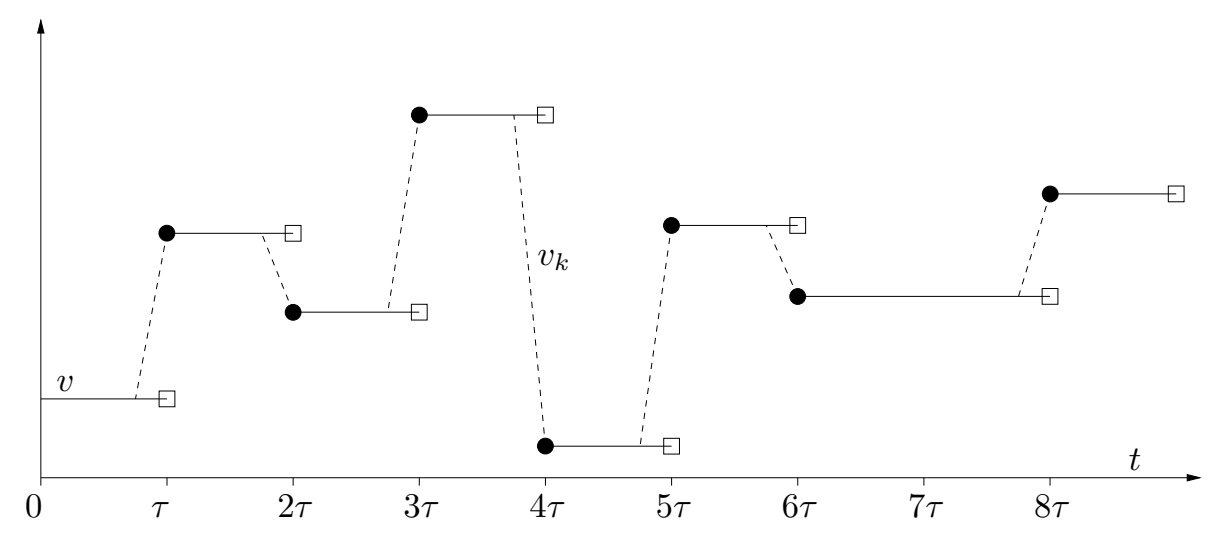

FIG. 5. Example of $v \in \mathcal{S}_{\tau}$ and its approximation $v_{k}$.

Proposition 4.4. Let $\Phi: C_{\mathrm{pm}}\left(\mathbb{R}_{+}, \mathbb{R}\right) \rightarrow C\left(\mathbb{R}_{+}, \mathbb{R}\right)$ be a hysteresis operator. Then

$$
\left(\Phi_{e}(v)\right)(t)=\lim _{k \rightarrow \infty}\left(\Phi\left(v_{k}\right)\right)(t) \quad \forall v \in \mathcal{S}_{\tau}, \quad \forall t \in \mathbb{R}_{+} .
$$

Proof. Let $t \in \mathbb{R}_{+}$, and let $m \in \mathbb{Z}_{+}$and $s \in[0, \tau)$ be such that $t=m \tau+s$. Choose $k$ sufficiently large so that

$$
t-m \tau=s<\left(1-\varepsilon_{k}\right) \tau .
$$

Then, using Proposition 4.3, part (5), we have

$$
\left(\Phi_{e}(v)\right)(t)=\left(\Phi_{e}(v)\right)(m \tau)=\varphi_{e}\left(\mathbf{Q}_{m \tau}^{c} v\right)=\varphi\left(\left(P_{\tau} \circ R_{e}\right)\left(\mathbf{Q}_{m \tau}^{c} v\right)\right) .
$$

Now $R_{e}\left(\mathbf{Q}_{m \tau}^{c} v\right)=R\left(\mathbf{Q}_{m \tau}^{c} v_{k}\right)=R_{e}\left(\mathbf{Q}_{m \tau}^{c} v_{k}\right)$, and hence, using (4.6), (4.7), Proposition 4.2, part (1), and Proposition 4.3, we obtain for all sufficiently large $k$

$$
\begin{aligned}
\left(\Phi_{e}(v)\right)(t) & =\varphi\left(\left(P_{\tau} \circ R_{e}\right)\left(\mathbf{Q}_{m \tau}^{c} v_{k}\right)\right)=\varphi_{e}\left(\mathbf{Q}_{m \tau}^{c} v_{k}\right)=\left(\Phi_{e}\left(v_{k}\right)\right)(m \tau) \\
& =\left(\Phi\left(v_{k}\right)\right)(m \tau)=\left(\Phi\left(v_{k}\right)\right)(t) . \quad \square
\end{aligned}
$$

A function $v \in F\left(\mathbb{R}_{+}, \mathbb{R}\right)$ is called ultimately nondecreasing if there exists $T \in \mathbb{R}_{+}$ such that $v$ is nondecreasing on $[T, \infty)$. Let $\mathcal{F} \subset F\left(\mathbb{R}_{+}, \mathbb{R}\right), \mathcal{F} \neq \emptyset$. The numerical value set NVS $\Phi$ of an operator $\Phi: \mathcal{F} \rightarrow F\left(\mathbb{R}_{+}, \mathbb{R}\right)$ is defined by

$$
\operatorname{NVS} \Phi:=\left\{(\Phi(v))(t) \mid v \in \mathcal{F}, t \in \mathbb{R}_{+}\right\} .
$$

For $\alpha \geq 0, u \in C_{\mathrm{pm}}([0, \alpha], \mathbb{R})$, and $\delta_{1}, \delta_{2}>0$, we define $\mathcal{C}_{\mathrm{pm}}\left(u ; \delta_{1}, \delta_{2}\right)$ to be the set of all $v \in C_{\mathrm{pm}}\left(\mathbb{R}_{+}, \mathbb{R}\right)$ such that

$$
v(t)=u(t) \forall t \in[0, \alpha] \quad \text { and } \quad|v(t)-u(\alpha)| \leq \delta_{1} \forall t \in\left[\alpha, \alpha+\delta_{2}\right] .
$$

We introduce the following seven assumptions on the operator $\Phi: C_{\mathrm{pm}}\left(\mathbb{R}_{+}, \mathbb{R}\right) \rightarrow$ $C\left(\mathbb{R}_{+}, \mathbb{R}\right)$ :

(C1) $\Phi$ is causal;

(C2) $\Phi$ is rate independent;

(C3) $\Phi\left(A C\left(\mathbb{R}_{+}, \mathbb{R}\right) \cap C_{\mathrm{pm}}\left(\mathbb{R}_{+}, \mathbb{R}\right)\right) \subset A C\left(\mathbb{R}_{+}, \mathbb{R}\right)$; 
(C4) $\Phi$ is monotone in the sense that, for all $v \in A C\left(\mathbb{R}_{+}, \mathbb{R}\right) \cap C_{\mathrm{pm}}\left(\mathbb{R}_{+}, \mathbb{R}\right)$ with $\Phi(v) \in A C\left(\mathbb{R}_{+}, \mathbb{R}\right)$,

$$
\frac{d}{d t}(\Phi(v))(t) \dot{v}(t) \geq 0, \quad \text { a.e. } t \in \mathbb{R}_{+} ;
$$

(C5) there exists $\lambda>0$ such that for all $\alpha \in \mathbb{R}_{+}, u \in C_{\mathrm{pm}}([0, \alpha], \mathbb{R})$, there exist numbers $\delta_{1}, \delta_{2}>0$ such that for all $v, w \in \mathcal{C}_{\mathrm{pm}}\left(u ; \delta_{1}, \delta_{2}\right)$

$$
\sup _{t \in\left[\alpha, \alpha+\delta_{2}\right]}|(\Phi(v))(t)-(\Phi(w))(t)| \leq \lambda \sup _{t \in\left[\alpha, \alpha+\delta_{2}\right]}|v(t)-w(t)| ;
$$

(C6) if $v \in C_{\mathrm{pm}}\left(\mathbb{R}_{+}, \mathbb{R}\right)$ is ultimately nondecreasing and $\lim _{t \rightarrow \infty} v(t)=\infty$, then $\Phi(v)(t)$ and $\Phi(-v)(t)$ converge to sup NVS $\Phi$ and inf NVS $\Phi$, respectively, as $t \rightarrow \infty$;

(C7) if, for $v \in C_{\mathrm{pm}}\left(\mathbb{R}_{+}, \mathbb{R}\right), L:=\lim _{t \rightarrow \infty}(\Phi(v))(t)$ exists with $L \in \operatorname{int}$ NVS $\Phi$, then $v$ is bounded.

REMARK 4.5. We note that if $\Phi$ satisfies (C1) and (C6), then NVS $\Phi$ is an interval.

If $\Phi: C_{\mathrm{pm}}\left(\mathbb{R}_{+}, \mathbb{R}\right) \rightarrow C\left(\mathbb{R}_{+}, \mathbb{R}\right)$ satisfies (C5), then any number $l>0$ such that (C5) holds for $\lambda=l$ is called a Lipschitz constant of $\Phi$.

Definition 4.6. Let $\lambda>0$. The set of all operators $\Phi: C_{\mathrm{pm}}\left(\mathbb{R}_{+}, \mathbb{R}\right) \rightarrow C\left(\mathbb{R}_{+}, \mathbb{R}\right)$ satisfying $(\mathrm{C} 1)-(\mathrm{C} 7)$ and having Lipschitz constant $\lambda$ is denoted by $\mathrm{C}(\lambda)$.

We consider four examples of hysteresis operators which satisfy $(\mathrm{C} 1)-(\mathrm{C} 7)$.

Static nonlinearities. For $f \in F(\mathbb{R}, \mathbb{R})$, the corresponding static nonlinearity

$$
F\left(\mathbb{R}_{+}, \mathbb{R}\right) \rightarrow F\left(\mathbb{R}_{+}, \mathbb{R}\right), \quad u \mapsto f \circ u
$$

is in $\mathcal{C}(\lambda)$, provided that $f$ is nondecreasing and globally Lipschitz with Lipschitz constant $\lambda$.

Relay. Relay (also called passive or positive) hysteresis, has been discussed in a mathematically rigorous context in a number of references; see, for example, [11] and [20]. To give a formal definition of relay, let $a_{1}, a_{2} \in \mathbb{R}$ with $a_{1}<a_{2}$, and let $\rho_{1}:\left[a_{1}, \infty\right) \rightarrow \mathbb{R}$ and $\rho_{2}:\left(-\infty, a_{2}\right] \rightarrow \mathbb{R}$ be nondecreasing and globally Lipschitz (both with Lipschitz constant $\lambda>0)$ and such that $\rho_{1}\left(a_{1}\right)=\rho_{2}\left(a_{1}\right)$ and $\rho_{1}\left(a_{2}\right)=\rho_{2}\left(a_{2}\right)$. For $v \in C_{\mathrm{pm}}\left(\mathbb{R}_{+}, \mathbb{R}\right)$ and $t \geq 0$ define

$$
S(v, t):=v^{-1}\left(\left\{a_{1}, a_{2}\right\}\right) \cap[0, t], \quad \tau(v, t):= \begin{cases}\max S(v, t) & \text { if } S(v, t) \neq \emptyset, \\ -1 & \text { if } S(v, t)=\emptyset .\end{cases}
$$

Following Macki, Nistri, and Zecca [20], for each $\xi \in \mathbb{R}$, we define an operator $\mathcal{R}_{\xi}$ : $C_{\mathrm{pm}}\left(\mathbb{R}_{+}, \mathbb{R}\right) \rightarrow C\left(\mathbb{R}_{+}, \mathbb{R}\right)$ by

$$
\left(\mathcal{R}_{\xi}(v)\right)(t)= \begin{cases}\rho_{2}(v(t)) & \text { if } v(t) \leq a_{1}, \\ \rho_{1}(v(t)) & \text { if } v(t) \geq a_{2} \\ \rho_{2}(v(t)) & \text { if } v(t) \in\left(a_{1}, a_{2}\right), \tau(v, t) \neq-1, \text { and } v(\tau(v, t))=a_{1}, \\ \rho_{1}(v(t)) & \text { if } v(t) \in\left(a_{1}, a_{2}\right), \tau(v, t) \neq-1, \text { and } v(\tau(v, t))=a_{2}, \\ \rho_{1}(v(t)) & \text { if } v(t) \in\left(a_{1}, a_{2}\right), \tau(v, t)=-1, \text { and } \xi>0 \\ \rho_{2}(v(t)) & \text { if } v(t) \in\left(a_{1}, a_{2}\right), \tau(v, t)=-1, \text { and } \xi \leq 0\end{cases}
$$

The number $\xi$ plays the role of an "initial state" which determines the output value $\left(\mathcal{R}_{\xi}(v)\right)(t)$ if $v(s) \in\left(a_{1}, a_{2}\right)$ for all $s \in[0, t]$. The relay hysteresis operator $\mathcal{R}_{\xi}$ is 


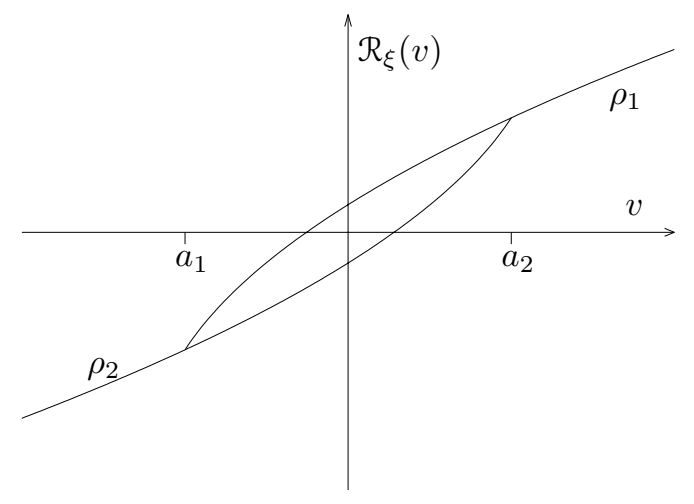

FIG. 6. Relay hysteresis.

illustrated in Figure 6. It is trivial that $\mathcal{R}_{\xi}$ is causal and rate independent (i.e., (C1) and (C2) hold). From [11] we know that $\mathcal{R}_{\xi}$ satisfies $(\mathrm{C} 3)-(\mathrm{C} 7)$ and that $\lambda$ is a Lipschitz constant of $\mathcal{R}_{\xi}$. It follows that $\mathcal{R}_{\xi} \in \mathcal{C}(\lambda)$.

Backlash. A discussion of the continuous-time backlash operator (also called the play operator) can be found in a number of references; see, for example, [1], [2], [8], and [11]. For all $h \in \mathbb{R}_{+}$and all $\xi \in \mathbb{R}$, we define the continuous-time backlash operator $\mathcal{B}_{h, \xi}: C_{\mathrm{pm}}\left(\mathbb{R}_{+}, \mathbb{R}\right) \rightarrow C\left(\mathbb{R}_{+}, \mathbb{R}\right)$ by

$$
\left(\mathcal{B}_{h, \xi}(v)\right)(t)= \begin{cases}b_{h}(v(0), \xi) & \text { for } t=0, \\ b_{h}\left(v(t),\left(\mathcal{B}_{h, \xi}(v)\right)\left(t_{i}\right)\right) & \text { for } t_{i}<t \leq t_{i+1}, \quad i \in \mathbb{Z}_{+},\end{cases}
$$

where the function $b_{h}: \mathbb{R}^{2} \rightarrow \mathbb{R}$ is given by (2.1) and $0=t_{0}<t_{1}<t_{2}<\cdots$ is such that $\lim _{n \rightarrow \infty} t_{n}=\infty$ and $v$ is monotone on each interval $\left(t_{i}, t_{i+1}\right)$. We remark that $\xi$ plays the role of an "initial state." It is not difficult to show that the definition is independent of the choice of the partition $\left(t_{i}\right)$; see [11]. The diagram illustrating how $\mathcal{B}_{h, \xi}$ acts is the same as in the discrete-time case; see Figure 3 . It is trivial that $\mathcal{B}_{h, \xi}$ is causal (i.e., $\mathcal{B}_{h, \xi}$ satisfies $(\mathrm{C} 1)$ ). Furthermore, it is well known and easy to check that $\mathcal{B}_{h, \xi}$ is rate independent (i.e., $\mathcal{B}_{h, \xi}$ satisfies (C2)). From [11] we know that $\mathcal{B}_{h, \xi}$ satisfies (C3)-(C7) and that $\lambda=1$ is a Lipschitz constant of $\mathcal{B}_{h, \xi}$. Therefore, we may conclude that $\mathcal{B}_{h, \xi} \in \mathcal{C}(1)$.

Elastic-plastic. The elastic-plastic operator (also called the stop operator) has been discussed in a mathematically rigorous context in a number of references; see, for example, [1], [2], [8], and [11]. To give a formal definition of the elastic-plastic operator, define for each $h \in \mathbb{R}_{+}$the function $e_{h}: \mathbb{R} \rightarrow \mathbb{R}$ by

$$
e_{h}(w)=\min \{h, \max \{-h, w\}\} .
$$

For all $h \in \mathbb{R}_{+}$and all $\xi \in \mathbb{R}$, we define an operator $\mathcal{E}_{h, \xi}: C_{\mathrm{pm}}\left(\mathbb{R}_{+}, \mathbb{R}\right) \rightarrow C\left(\mathbb{R}_{+}, \mathbb{R}\right)$ by

$$
\left(\mathcal{E}_{h, \xi}(v)\right)(t)= \begin{cases}e_{h}(v(0)-\xi) & \text { for } t=0, \\ e_{h}\left(v(t)-v\left(t_{i}\right)+\left(\mathcal{E}_{h, \xi}(v)\right)\left(t_{i}\right)\right) & \text { for } t_{i}<t \leq t_{i+1}, \quad i \in \mathbb{Z}_{+},\end{cases}
$$

where $0=t_{0}<t_{1}<t_{2}<\cdots$ is such that $\lim _{n \rightarrow \infty} t_{n}=\infty$ and $v$ is monotone on each interval $\left(t_{i}, t_{i+1}\right)$. Again, $\xi$ plays the role of an "initial state." The backlash and 


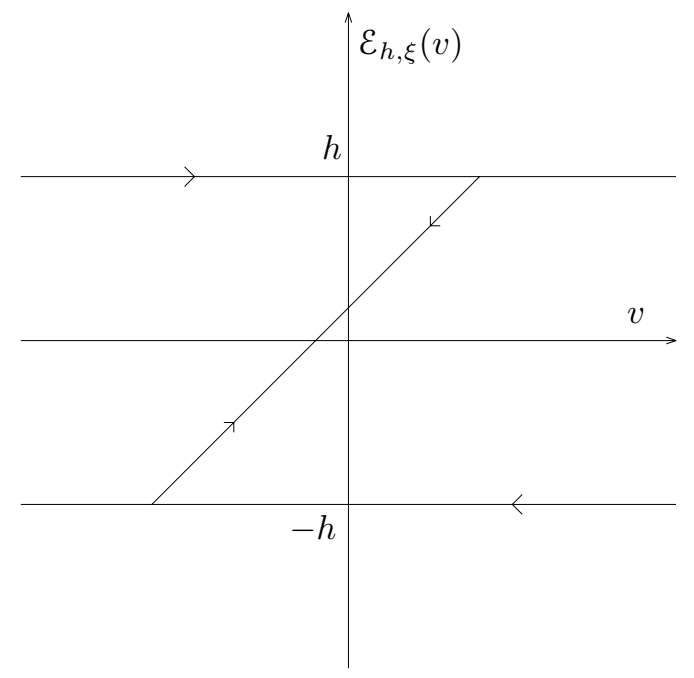

FIG. 7. Elastic-plastic hysteresis.

elastic-plastic operators are closely related:

$$
\mathcal{E}_{h, \xi}(v)+\mathcal{B}_{h, \xi}(v)=v \quad \forall v \in C_{\mathrm{pm}}\left(\mathbb{R}_{+}, \mathbb{R}\right) ;
$$

see $\left[2\right.$, p. 44]. The elastic-plastic operator $\mathcal{E}_{h, \xi}$ is illustrated in Figure 7. Since $\mathcal{B}_{h, \xi}$ is causal and rate independent, it follows from (4.8) that $\varepsilon_{h, \xi}$ is causal and rate independent; i.e., (C1) and (C2) hold. From [11] we know that $\varepsilon_{h, \xi}$ satisfies (C3)-(C7) and that $\lambda=2$ is a Lipschitz constant of $\mathcal{E}_{h, \xi}$. Therefore, $\mathcal{E}_{h, \xi} \in \mathcal{C}(2)$.

We remark that a large class of Prandtl and Preisach hysteresis operators satisfy (C1)-(C7); see [11] for details.

The following lemma will be needed later in this section.

Lemma 4.7. Let $\Phi \in \mathcal{C}(\lambda)$; then for every $v \in C_{\mathrm{pm}}\left(\mathbb{R}_{+}, \mathbb{R}\right) \cap A C\left(\mathbb{R}_{+}, \mathbb{R}\right)$ and $t_{2}>t_{1} \geq 0$, there exists a constant $\eta \in[0, \lambda]$ such that

$v$ affine linear on $\left[t_{1}, t_{2}\right] \quad \Longrightarrow \quad(\Phi(v))\left(t_{2}\right)-(\Phi(v))\left(t_{1}\right)=\eta\left(v\left(t_{2}\right)-v\left(t_{1}\right)\right)$.

Proof. Let $\Phi \in \mathcal{C}(\lambda), v \in C_{\mathrm{pm}}\left(\mathbb{R}_{+}, \mathbb{R}\right) \cap A C\left(\mathbb{R}_{+}, \mathbb{R}\right)$, and $t_{2}>t_{1} \geq 0$, and assume that $v$ is affine linear on $\left[t_{1}, t_{2}\right]$. By Proposition 4.2, part (1) and [11, Lemma 3.2, part (c)] there exists a measurable function $d: \mathbb{R}_{+} \rightarrow[0, \lambda]$ such that

$$
(\Phi(v))\left(t_{2}\right)-(\Phi(v))\left(t_{1}\right)=\int_{t_{1}}^{t_{2}} d(t) \dot{v}(t) d t .
$$

Since $v$ is affine linear on $\left[t_{1}, t_{2}\right], \dot{v} \equiv\left(v\left(t_{2}\right)-v\left(t_{1}\right)\right) /\left(t_{2}-t_{1}\right)$ on $\left(t_{1}, t_{2}\right)$. Combining this with (4.9) gives

$$
(\Phi(v))\left(t_{2}\right)-(\Phi(v))\left(t_{1}\right)=\frac{v\left(t_{2}\right)-v\left(t_{1}\right)}{t_{2}-t_{1}} \int_{t_{1}}^{t_{2}} d(t) d t=\eta\left(v\left(t_{2}\right)-v\left(t_{1}\right)\right),
$$

where $\eta=1 /\left(t_{2}-t_{1}\right) \int_{t_{1}}^{t_{2}} d(t) d t \in[0, \lambda]$.

In the following section, we want to apply the discrete-time result of section 3 to the sampled-data low-gain integral control problem. Therefore, we need to investigate 
the properties of the discretization of a given $\Phi^{c} \in \mathcal{C}(\lambda)$ obtained by standard hold and sampling operations. To this end, define the hold operator $H: F\left(\mathbb{Z}_{+}, \mathbb{R}\right) \rightarrow \mathcal{S}_{\tau}$ by

$$
(H u)(n \tau+t)=u(n) \quad \forall u \in F\left(\mathbb{Z}_{+}, \mathbb{R}\right), \quad \forall n \in \mathbb{Z}_{+}, \quad \forall t \in[0, \tau),
$$

and the sampling operator $S: P C\left(\mathbb{R}_{+}, \mathbb{R}\right) \rightarrow F\left(\mathbb{Z}_{+}, \mathbb{R}\right)$ by

$$
(S v)(n)=v(n \tau) \quad \forall v \in P C\left(\mathbb{R}_{+}, \mathbb{R}\right), \quad \forall n \in \mathbb{Z}_{+},
$$

where $\tau>0$ denotes the sampling period.

Let $\Phi^{c}: C_{\mathrm{pm}}\left(\mathbb{R}_{+}, \mathbb{R}\right) \rightarrow C\left(\mathbb{R}_{+}, \mathbb{R}\right)$ be a continuous-time hysteresis operator. We define a discrete-time operator $\Phi: F\left(\mathbb{Z}_{+}, \mathbb{R}\right) \rightarrow F\left(\mathbb{Z}_{+}, \mathbb{R}\right)$ by

$$
\Phi:=S \Phi_{e}^{c} H
$$

where $\Phi_{e}^{c}$ denotes the extension of $\Phi^{c}$ to $N P C_{\mathrm{pm}}\left(\mathbb{R}_{+}, \mathbb{R}\right)$ given by (4.5). We remark that as a simple consequence of the rate independence of $\Phi_{e}^{c}$ (cf. Proposition 4.3) the definition of $\Phi$ is independent of the choice of the sampling period $\tau$. For $v \in \mathcal{S}_{\tau}$, we have $H S v=v$, and so, by Proposition 4.3, part (5),

$$
\Phi_{e}^{c} H=H \Phi .
$$

The proof of the following result can be found in [10].

LEMma 4.8. Let $\Phi^{c}: C_{\mathrm{pm}}\left(\mathbb{R}_{+}, \mathbb{R}\right) \rightarrow C\left(\mathbb{R}_{+}, \mathbb{R}\right)$ be a hysteresis operator, and define the operator $\Phi: F\left(\mathbb{Z}_{+}, \mathbb{R}\right) \rightarrow F\left(\mathbb{Z}_{+}, \mathbb{R}\right)$ by (4.12). Then

$$
(\Phi(u))(n)=\left(\Phi^{c}\left(P_{\tau} u\right)\right)(n \tau) \quad \forall u \in F\left(\mathbb{Z}_{+}, \mathbb{R}\right), \quad \forall n \in \mathbb{Z}_{+},
$$

and $\operatorname{NVS} \Phi=\operatorname{NVS} \Phi^{c}$.

The following proposition is the main result of this section.

Proposition 4.9. Let $\Phi^{c} \in \mathcal{C}(\lambda)$, and define $\Phi: F\left(\mathbb{Z}_{+}, \mathbb{R}\right) \rightarrow F\left(\mathbb{Z}_{+}, \mathbb{R}\right)$ by (4.12). Then $\Phi \in \mathcal{D}(\lambda)$.

Proof. Note that (D1) (causality) follows from (4.14) and the causality of $\Phi^{c}$, while (D2) follows from Proposition 4.2, part (1) and (4.14). Furthermore, let $u \in F\left(\mathbb{Z}_{+}, \mathbb{R}\right)$, $n \in \mathbb{Z}_{+}$, and suppose that $u(n+1) \neq u(n)$. Then by (4.14) and Lemma 4.7 there exists a constant $\eta \in[0, \lambda]$ such that

$$
\begin{aligned}
(\Phi(u))(n+1)-(\Phi(u))(n) & =\left(\Phi^{c}\left(P_{\tau} u\right)\right)((n+1) \tau)-\left(\Phi^{c}\left(P_{\tau} u\right)\right)(n \tau) \\
& =\eta\left[\left(P_{\tau} u\right)((n+1) \tau)-\left(P_{\tau} u\right)(n \tau)\right] \\
& =\eta[u(n+1)-u(n)],
\end{aligned}
$$

and thus (D3) holds. Since NVS $\Phi=\operatorname{NVS} \Phi^{c}$ (by Lemma 4.8) and $\Phi^{c}$ satisfies (C6), (D4) follows from an application of (4.14). Finally, to show that (D5) is satisfied, let $u \in F\left(\mathbb{Z}_{+}, \mathbb{R}\right)$ be such that $\lim _{n \rightarrow \infty}(\Phi(u))(n)$ exists and

$$
L:=\lim _{n \rightarrow \infty}(\Phi(u))(n) \in \operatorname{int}(\operatorname{clos}(\operatorname{NVS} \Phi)) .
$$

By (4.14),

$$
\lim _{n \rightarrow \infty}\left(\Phi^{c}\left(P_{\tau} u\right)\right)(n \tau)=L
$$


Clearly $P_{\tau} u$ is monotone on $[n \tau,(n+1) \tau]$ for each $n \in \mathbb{Z}_{+}$, and therefore, by the fact that $\Phi^{c}$ satisfies $(\mathrm{C} 4), \Phi^{c}\left(P_{\tau} u\right)$ is monotone on $[n \tau,(n+1) \tau]$ for each $n \in \mathbb{Z}_{+}$. Combining this with (4.16) shows that

$$
\lim _{t \rightarrow \infty}\left(\Phi^{c}\left(P_{\tau} u\right)\right)(t)=L .
$$

By Remark 4.5, $\mathrm{NVS}^{\mathrm{c}}$ is an interval; since, by Lemma $4.8, \operatorname{NVS} \Phi=\mathrm{NVS} \Phi^{\mathrm{c}}$, it follows from (4.15) that $L \in \operatorname{int}\left(\operatorname{NVS} \Phi^{\mathrm{c}}\right)$. Now $\Phi^{c}$ satisfies $(\mathrm{C} 7)$, and so we may conclude that $P_{\tau} u$ and thus $u$ are bounded.

5. Sampled-data integral control in the presence of input hysteresis. In the following, the underlying linear system is assumed to be a single-input, singleoutput, continuous-time, regular system $\Sigma$ (for details on regular systems see Weiss [25], [26], [27], [28]) with state-space $X$ (a real Hilbert space) and with generating operators $\left(A^{c}, B^{c}, C^{c}, D^{c}\right)$. This means, in particular, that $A^{c}$ generates a strongly continuous semigroup $\mathbf{T}=\left(\mathbf{T}_{t}\right)_{t \geq 0}, C^{c} \in L\left(X_{1}, \mathbb{R}\right)$ is an admissible observation operator for $\mathbf{T}$, and $B^{c} \in L\left(\mathbb{R}, X_{-1}\right)$ is an admissible control operator for $\mathbf{T}$. Here $X_{1}$ denotes the domain of $A^{c}$ endowed with the graph norm, and $X_{-1}$ denotes the completion of $X$ with respect to the norm $\|x\|_{-1}=\left\|\left(s_{0} I-A^{c}\right)^{-1} x\right\|$, where $s_{0}$ is any fixed element in the resolvent set of $A^{c}$. The norm on $X$ is denoted by $\|\cdot\|$, while $\|\cdot\|_{1}$ and $\|\cdot\|_{-1}$ denote the norms on $X_{1}$ and $X_{-1}$, respectively. Then $X_{1} \hookrightarrow X \hookrightarrow X_{-1}$, and $\mathbf{T}$ restricts (respectively, extends) to a strongly continuous semigroup on $X_{1}$ (respectively, $X_{-1}$ ). The exponential growth constant

$$
\omega(\mathbf{T}):=\lim _{t \rightarrow \infty} \frac{1}{t} \ln \left\|\mathbf{T}_{t}\right\|
$$

is the same on all three spaces. The generator of $\mathbf{T}$ on $X_{-1}$ is an extension of $A^{c}$ to $X$ (which is bounded as an operator from $X$ to $X_{-1}$ ). We shall use the same symbol $\mathbf{T}$ (respectively, $A^{c}$ ) for the original semigroup (respectively, its generator) and the associated restrictions and extensions. With this convention, we may write $A^{c} \in L\left(X, X_{-1}\right)$. Considered as a generator on $X_{-1}$, the domain of $A^{c}$ is $X$.

We regard a regular system $\Sigma$ as synonymous with its generating operators and simply write $\Sigma=\left(A^{c}, B^{c}, C^{c}, D^{c}\right)$. The regular system is said to be exponentially stable if the semigroup $\mathbf{T}$ is exponentially stable, that is, $\omega(\mathbf{T})<0$. The control operator $B^{c}$ (respectively, observation operator $C^{c}$ ) is said to be bounded if $B^{c} \in$ $L(\mathbb{R}, X)$ (respectively, $C^{c} \in L(X, \mathbb{R})$ ); otherwise, $B^{c}$ (respectively, $C^{c}$ ) is said to be unbounded. In terms of the generating operators $\left(A^{c}, B^{c}, C^{c}, D^{c}\right)$, the transfer function $\mathbf{G}^{c}(s)$ can be expressed as

$$
\mathbf{G}^{c}(s)=C_{L}^{c}\left(s I-A^{c}\right)^{-1} B^{c}+D^{c},
$$

where $C_{L}^{c}$ denotes the so-called Lebesgue extension of $C^{c}$. The transfer function $\mathbf{G}^{c}(s)$ is bounded and holomorphic in any half-plane $\operatorname{Re} s>\alpha$ with $\alpha>\omega(\mathbf{T})$. Moreover,

$$
\lim _{s \rightarrow \infty, s \in \mathbb{R}} \mathbf{G}^{c}(s)=D^{c} .
$$

For any $x_{0} \in X$ and $u^{c} \in L_{\text {loc }}^{2}\left(\mathbb{R}_{+}, \mathbb{R}\right)$, the state and output functions $x^{c}(\cdot)$ and $y^{c}(\cdot)$, respectively, satisfy the equations

$$
\begin{aligned}
\dot{x}^{c}(t) & =A^{c} x^{c}(t)+B^{c} u^{c}(t), \quad x^{c}(0)=x_{0}, \\
y^{c}(t) & =C_{L}^{c} x^{c}(t)+D^{c} u^{c}(t)
\end{aligned}
$$


for almost all $t \geq 0$. The derivative on the left-hand side of (5.1a), of course, has to be understood in $X_{-1}$. In other words, if we consider the initial-value problem (5.1a) in the space $X_{-1}$, then for any $x_{0} \in X$ and $u^{c} \in L_{\text {loc }}^{2}\left(\mathbb{R}_{+}, \mathbb{R}\right),(5.1 \mathrm{a})$ has unique strong solution (in the sense of Pazy [24, p. 109]) given by the variation of parameters formula

$$
x^{c}(t)=\mathbf{T}_{t} x_{0}+\int_{0}^{t} \mathbf{T}_{t-\tau} B^{c} u^{c}(\tau) d \tau .
$$

For future reference we state the following lemma, the proof of which can be found in $[15]$.

Lemma 5.1. Assume that $\mathbf{T}$ is exponentially stable and that $B^{c} \in L\left(\mathbb{R}, X_{-1}\right)$ is an admissible control operator for $\mathbf{T}$. If $u^{c} \in L^{\infty}\left(\mathbb{R}_{+}, \mathbb{R}\right)$ is such that $\lim _{t \rightarrow \infty} u^{c}(t)=u_{\infty}$ exists, then for all $x_{0} \in X$ the state trajectory $x^{c}$ given by (5.2) satisfies

$$
\lim _{t \rightarrow \infty}\left\|x^{c}(t)+\left(A^{c}\right)^{-1} B^{c} u_{\infty}\right\|=0 .
$$

The aim in this section is to show that for an exponentially stable, regular, linear, infinite-dimensional, continuous-time, single-input, single-output plant with transfer function $\mathbf{G}^{c}(s)$, subject to a continuous-time dynamic input nonlinearity $\Phi^{c} \in \mathcal{C}(\lambda)$, the output $y^{c}(t)$ of the sampled-data closed-loop system, shown in Figure 2, converges to the reference value $r$ as $t \rightarrow \infty$, provided that $\mathbf{G}^{c}(0)>0, r$ is feasible in some natural sense, and $k>0$ is sufficiently small.

Let $u \in F\left(\mathbb{Z}_{+}, \mathbb{R}\right)$, and apply the continuous-time signal

$$
u^{c}=H u
$$

(where $H$ is the standard hold operator defined in (4.10)) to the continuous-time system given by (5.1). Then the state $x^{c}(n \tau+t)$ satisfies

$$
x^{c}(n \tau+t)=\mathbf{T}_{t} x^{c}(n \tau)+\left(\mathbf{T}_{t}-I\right)\left(A^{c}\right)^{-1} B^{c} u(n) \quad \forall n \in \mathbb{Z}_{+}, \quad \forall t \in[0, \tau) .
$$

Accordingly, we define $x: \mathbb{Z}_{+} \rightarrow X$ by

$$
x(n)=x^{c}(n \tau) .
$$

Clearly, $\mathbf{T}_{\tau} \in L(X)$ and $\left(\mathbf{T}_{\tau}-I\right)\left(A^{c}\right)^{-1} B^{c} \in L(\mathbb{R}, X)$ define appropriate state-space operators for the state evolution of the discretization of (5.1a). However, in general, regularity guarantees only that $y^{c} \in L_{\text {loc }}^{2}\left(\mathbb{R}_{+}, \mathbb{R}\right)$ so that, even with piecewise constant input functions, standard sampling of the output is not defined. Moreover, even if the output function is continuous (in which case standard sampling is defined), in general the resulting discrete-time system will not have a bounded observation operator. We therefore distinguish two cases: bounded and unbounded continuous-time observation.

Bounded observation. Assume that $C^{c}=C_{L}^{c} \in L(X, \mathbb{R})$. If $x_{0} \in X$ and $u^{c}$ is given by (5.3), then the output $y^{c}$ given by $(5.1 \mathrm{~b})$ is piecewise continuous, the discontinuities being at $n \tau$. It is clear that $y^{c}$ is right-continuous at $n \tau$ for all $n \in \mathbb{Z}_{+}$. We define

$$
y:=S y^{c}
$$

(where $S$ is the standard sampling operator defined in (4.11)) and

$$
\left(\begin{array}{cc}
A & B \\
C & D
\end{array}\right):=\left(\begin{array}{cc}
\mathbf{T}_{\tau} & \left(\mathbf{T}_{\tau}-I\right)\left(A^{c}\right)^{-1} B^{c} \\
C^{c} & D^{c}
\end{array}\right) .
$$


The proof of the following proposition is an immediate consequence of Proposition 4.1 in [16].

Proposition 5.2. Suppose that $\mathbf{T}_{t}$ is exponentially stable and that the observation operator $C^{c}$ is bounded. Let $\tau>0$ and $u \in F\left(\mathbb{Z}_{+}, \mathbb{R}\right)$. If $u^{c}$ given by (5.3) is applied to (5.1), then $x$ and $y$ given by (5.5) and (5.6), respectively, satisfy (3.1), where $(A, B, C, D)$ is given by (5.7). Moreover, $A$ is power-stable, and, setting $\mathbf{G}(z)=C(z I-A)^{-1} B+D$, we have that

$$
\mathbf{G}(1)=C(I-A)^{-1} B+D=\mathbf{G}^{c}(0) .
$$

Let $\Phi^{c} \in \mathcal{C}(\lambda)$, and let $\Phi_{e}^{c}$ denote the extension of $\Phi^{c}$ to $N P C_{\mathrm{pm}}\left(\mathbb{R}_{+}, \mathbb{R}\right)$ given by (4.5). Consider the continuous-time system (5.1) with continuous-time input nonlinearity $\Phi_{e}^{c}$,

$$
\begin{aligned}
& \dot{x}^{c}=A^{c} x^{c}+B^{c} \Phi_{e}^{c}\left(u^{c}\right), \quad x^{c}(0)=x_{0} \in X, \\
& y^{c}=C_{L}^{c} x^{c}+D^{c} \Phi_{e}^{c}\left(u^{c}\right),
\end{aligned}
$$

controlled by the sampled-data integrator

$$
\begin{aligned}
u^{c}(t) & =u(n) \quad \text { for } t \in[n \tau,(n+1) \tau), \quad n \in \mathbb{Z}_{+}, \\
y(n) & =y^{c}(n \tau), \quad n \in \mathbb{Z}_{+}, \\
u(n+1) & =u(n)+k(r-y(n)), \quad u(0)=u_{0} \in \mathbb{R}, \quad n \in \mathbb{Z}_{+} .
\end{aligned}
$$

Theorem 5.3. Let $\lambda>0$. Assume that $\Phi^{c} \in \mathcal{C}(\lambda), C^{c}$ is bounded, $\mathbf{T}_{t}$ is exponentially stable, $\mathbf{G}^{c}(0)>0$, and $r \in \mathbb{R}$ is such that $\tilde{r}^{c}:=r / \mathbf{G}^{c}(0) \in \operatorname{clos}\left(\operatorname{NVS} \Phi^{c}\right)$. Let $K>0$ be defined by (3.5), where $\mathbf{G}(z)=C(z I-A)^{-1} B+D$, with $(A, B, C, D)$ given by (5.7). Then, for all $k \in(0, K / \lambda)$ and all $\left(x_{0}, u_{0}\right) \in X \times \mathbb{R}$, the unique solution $\left(x^{c}(\cdot), u^{c}(\cdot)\right)$ of the closed-loop system given by (5.9) and (5.10) satisfies the following:

(1) $\lim _{t \rightarrow \infty}\left(\Phi_{e}^{c}\left(u^{c}\right)\right)(t)=\tilde{r}^{c}$;

(2) $\lim _{t \rightarrow \infty}\left\|x^{c}(t)+\left(A^{c}\right)^{-1} B^{c} \tilde{r}^{c}\right\|=0$;

(3) $\lim _{t \rightarrow \infty} y^{c}(t)=r$;

(4) if $\tilde{r}^{c} \in \operatorname{int}\left(\operatorname{NVS} \Phi^{c}\right)$, then $u^{c}$ is bounded.

Proof. Let $\left(x^{c}(\cdot), u^{c}(\cdot)\right)$ be the unique solution of the closed-loop system given by (5.9) and (5.10). Define $\Phi \in \mathcal{D}(\lambda)$ by (4.12), and so

$$
\left(\Phi_{e}^{c}\left(u^{c}\right)\right)(n \tau)=\left(\Phi_{e}^{c}(H u)\right)(n \tau)=(\Phi(u))(n) \quad \forall n \in \mathbb{Z}_{+} .
$$

Note that by Lemma 4.8

$$
\operatorname{NVS} \Phi=\operatorname{NVS} \Phi^{c}
$$

Defining $x \in F\left(\mathbb{Z}_{+}, \mathbb{R}\right)$ by $(5.5)$, it follows from Proposition 5.2 that $(x, u)$ satisfies the closed-loop discrete-time equations (3.3), where $(A, B, C, D)$ is given by (5.7). Therefore, using Theorem 3.1, Propositions 4.9 and 5.2, and (5.11), we see that for all $k \in(0, K / \lambda)$

$$
\lim _{n \rightarrow \infty}(\Phi(u))(n)=\tilde{r}^{c} .
$$

This implies that for all $k \in(0, K / \lambda), \lim _{t \rightarrow \infty}(H(\Phi(u)))(t)=\tilde{r}^{c}$, and so by (4.13), $\lim _{t \rightarrow \infty}\left(\Phi_{e}^{c}\left(u^{c}\right)\right)(t)=\tilde{r}^{c}$, which is statement (1). Statement (2) is a consequence of statement (1) and Lemma 5.1. Statement (3) follows easily from statements (1) and (2) and the boundedness of $C^{c}$. Finally, to prove statement (4), assume that $\tilde{r}^{c} \in \operatorname{int} \operatorname{NVS} \Phi^{c}$. Then, by (5.11), $\tilde{r}^{c} \in \operatorname{int} \operatorname{NVS} \Phi$. Boundedness of $u$ and thus boundedness of $u^{c}$ now follow immediately from (5.12) and the fact that (D5) holds for $\Phi$ (by Proposition 4.9). 
Unbounded observation. As mentioned earlier, in this case, we cannot define a sampled output via (5.6). Instead, we introduce a generalized sampling operation. In the following, let $w \in L^{2}([0, \tau], \mathbb{R})$ be a function satisfying the conditions

$$
\text { (i) } \int_{0}^{\tau} w(t) d t=1 \quad \text { and } \quad \text { (ii) } \int_{0}^{\tau} w(t) \mathbf{T}_{t} x d t \in X_{1} \quad \forall x \in X .
$$

While condition (5.13) (ii) is difficult to check for general $w$, it is easy to show (using integration by parts) that (5.13) (ii) holds if there exists a partition $0=t_{0}<t_{1}<$ $\cdots<t_{m}=\tau$ such that $\left.w\right|_{\left(t_{i-1}, t_{i}\right)} \in W^{1,1}\left(\left(t_{i-1}, t_{i}\right), \mathbb{R}\right)$ for $i=1,2, \ldots, m$.

We define a generalized sampling operation by

$$
y(n)=\int_{0}^{\tau} w(t) y^{c}(n \tau+t) d t \quad \forall n \in \mathbb{Z}_{+} .
$$

Introducing the linear operator

$$
L: X \rightarrow X_{1}, \quad x \mapsto \int_{0}^{\tau} w(t) \mathbf{T}_{t} x d t
$$

we define

$$
\left(\begin{array}{cc}
A & B \\
C & D
\end{array}\right):=\left(\begin{array}{cc}
\mathbf{T}_{\tau} & \left(\mathbf{T}_{\tau}-I\right)\left(A^{c}\right)^{-1} B^{c} \\
C^{c} L & C^{c} L\left(A^{c}\right)^{-1} B^{c}+\mathbf{G}^{c}(0)
\end{array}\right)
$$

The following result is an immediate consequence of Proposition 3.4 in [12].

Proposition 5.4. Suppose that $\mathbf{T}_{t}$ is exponentially stable. Let $\tau>0$ and $u \in$ $F\left(\mathbb{Z}_{+}, \mathbb{R}\right)$. If $u^{c}$ given by (5.3) is applied to (5.1), then $x$ and $y$ given by (5.5) and (5.14), respectively, satisfy (3.1), where $(A, B, C, D)$ is given by (5.15). Moreover, $A$ is power-stable, $C \in L(X, \mathbb{R})$, and, setting $\mathbf{G}(z)=C(z I-A)^{-1} B+D$, (5.8) is satisfied.

Consider the following sampled-data low-gain controller for (5.9):

$$
\begin{aligned}
u^{c}(t) & =u(n) \quad \text { for } t \in[n \tau,(n+1) \tau), \quad n \in \mathbb{Z}_{+}, \\
y(n) & =\int_{0}^{\tau} w(t) y^{c}(n \tau+t) d t, \quad n \in \mathbb{Z}_{+}, \\
u(n+1) & =u(n)+k(r-y(n)), \quad u(0)=u_{0} \in \mathbb{R}, \quad n \in \mathbb{Z}_{+} .
\end{aligned}
$$

Theorem 5.5. Let $\lambda>0$. Assume that $\Phi^{c} \in \mathcal{C}(\lambda), \mathfrak{L}^{-1}\left(\mathbf{G}^{c}\right)$ is a finite signed Borel measure on $\mathbb{R}_{+}, \mathbf{T}_{t}$ is exponentially stable, $\mathbf{G}^{c}(0)>0$, and $r \in \mathbb{R}$ is such that $\tilde{r}^{c}:=r / \mathbf{G}^{c}(0) \in \operatorname{clos}\left(\operatorname{NVS} \Phi^{c}\right)$. Let $K>0$ be defined by (3.5), where $\mathbf{G}(z)=$ $C(z I-A)^{-1} B+D$, with $(A, B, C, D)$ given by (5.15). Then, for all $k \in(0, K / \lambda)$ and all $\left(x_{0}, u_{0}\right) \in X \times \mathbb{R}$, the unique solution $\left(x^{c}(\cdot), u^{c}(\cdot)\right)$ of the closed-loop system given by (5.9) and (5.16) satisfies the following:

(1) $\lim _{t \rightarrow \infty}\left(\Phi_{e}^{c}\left(u^{c}\right)\right)(t)=\tilde{r}^{c}$;

(2) $\lim _{t \rightarrow \infty}\left\|x^{c}(t)+\left(A^{c}\right)^{-1} B^{c} \tilde{r}^{c}\right\|=0$;

(3) $\lim _{t \rightarrow \infty}\left[r-y^{c}(t)+C_{L}^{c} \mathbf{T}_{t} x_{0}\right]=0$;

(4) if $\tilde{r}^{c} \in \operatorname{int}\left(\operatorname{NVS} \Phi^{c}\right)$, then $u^{c}$ is bounded.

REMARK 5.6. (1) Since $C_{L}^{c} \mathbf{T}_{t} x_{0}$ converges exponentially to 0 as $t \rightarrow \infty$ for all $x_{0} \in X_{1}$, it follows from statement (3) that the error $e^{c}(t)=r-y^{c}(t)$ converges to 0 for all $x_{0} \in X_{1}$. If $C^{c}$ is bounded, then this statement is true for all $x_{0} \in X$. If $C^{c}$ is unbounded and $x_{0} \notin X_{1}$, then $e^{c}(t)$ does not necessarily converge to 0 as $t \rightarrow \infty$. 
However, it follows from the proof below that $e^{c}(t)$ is small for large $t$ in the sense that $e^{c}(t)=e_{1}^{c}(t)+e_{2}^{c}(t)$, where the function $e_{1}^{c}$ is bounded with $\lim _{t \rightarrow \infty} e_{1}^{c}(t)=0$ and the function $t \mapsto e_{2}^{c}(t) \exp (\alpha t)$ is in $L^{2}\left(\mathbb{R}_{+}, \mathbb{R}\right)$ for some $\alpha>0$.

(2) The assumption that $\mathfrak{L}^{-1}\left(\mathbf{G}^{c}\right)$ is a finite signed Borel measure on $\mathbb{R}_{+}$is not very restrictive and seems to be satisfied in all practical examples of exponentially stable regular systems. In particular, this assumption is satisfied if $B^{c}$ or $C^{c}$ is bounded (see [13, Lemma 2.3]).

Proof of Theorem 5.5. By using Proposition 5.4 instead of Proposition 5.2, all statements with the exception of (3) follow exactly as in the proof of Theorem 5.3. Due to the unboundedness of $C^{c}$, we cannot use statement (2) in order to show that $y^{c}(t)$ converges to $r$ as $t \rightarrow \infty$. However, we have

$$
y^{c}(t)=C_{L}^{c} \mathbf{T}_{t} x_{0}+\left(\mathfrak{L}^{-1}\left(\mathbf{G}^{c}\right) \star \Phi_{e}^{c}\left(u^{c}\right)\right)(t) .
$$

By assumption, $\mathfrak{L}^{-1}\left(\mathbf{G}^{c}\right)$ is a finite signed Borel measure, and since $\lim _{t \rightarrow \infty}\left(\Phi_{e}^{c}\left(u^{c}\right)\right)(t)$ $=\tilde{r}^{c}$ (by statement (1)), it follows from [5, Theorem 6.1, part (ii), p. 96] that

$$
\lim _{t \rightarrow \infty}\left[\mathfrak{L}^{-1}\left(\mathbf{G}^{c}\right) \star \Phi_{e}^{c}\left(u^{c}\right)\right](t)=\mathbf{G}^{c}(0) \tilde{r}^{c}=r .
$$

Combining this with (5.17) shows that statement (3) holds.

6. Example: Sampled-data control of a diffusion process with output delay subject to input hysteresis. Consider a diffusion process (with diffusion coefficient $\kappa>0$ and with Dirichlet boundary conditions) on the one-dimensional spatial domain $[0,1]$, with scalar nonlinear pointwise control action (applied at point $x_{1} \in(0,1)$, via an operator $\Phi^{c} \in \mathcal{C}(\lambda)$ ) and delayed (delay $T \geq 0$ ) scalar observation generated by a spatial averaging of the delayed state over an $\varepsilon$-neighborhood of a point $x_{2} \in(0,1)$ with $x_{2}>x_{1}$.

We formally write this single-input, single-output system as

$$
\begin{aligned}
z_{t}(t, x) & =\kappa z_{x x}(t, x)+\delta\left(x-x_{1}\right)\left(\Phi_{e}^{c}\left(u^{c}\right)\right)(t), \\
y^{c}(t) & =\frac{1}{2 \varepsilon} \int_{x_{2}-\varepsilon}^{x_{2}+\varepsilon} z(t-T, x) d x
\end{aligned}
$$

with boundary conditions

$$
z(t, 0)=0=z(t, 1) \quad \forall t>0 .
$$

For simplicity, we assume zero initial conditions

$$
z(t, x)=0 \quad \forall(t, x) \in[-T, 0] \times[0,1] .
$$

With input $\left(\Phi_{e}^{c}\left(u^{c}\right)\right)(\cdot)$ and output $y^{c}(\cdot)$, this example qualifies as a regular linear system with bounded observation and with transfer function given by

$\mathbf{G}^{c}(s)=\frac{e^{-s T} \sinh \left(x_{1} \sqrt{s / \kappa}\right)\left[\cosh \left(\left(1-x_{2}+\varepsilon\right) \sqrt{s / \kappa}\right)-\cosh \left(\left(1-x_{2}-\varepsilon\right) \sqrt{s / \kappa}\right)\right]}{2 \varepsilon s \sinh \sqrt{s / \kappa}}$.

Since the observation is bounded, we may sample the output using the standard sampling operation given by (5.6). Further analysis (invoking application of the maximum principle for the heat equation, which, for brevity, we omit here) confirms the physical intuition that the impulse response $\mathfrak{L}^{-1}\left(\mathbf{G}^{c}\right)$ is nonnegative-valued. Consequently, 


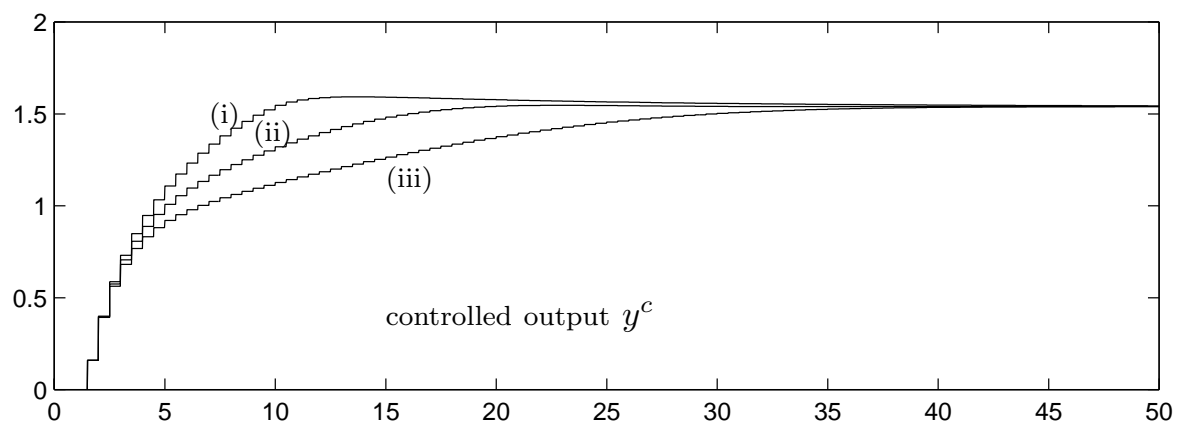

FiG. 8. Controlled output.

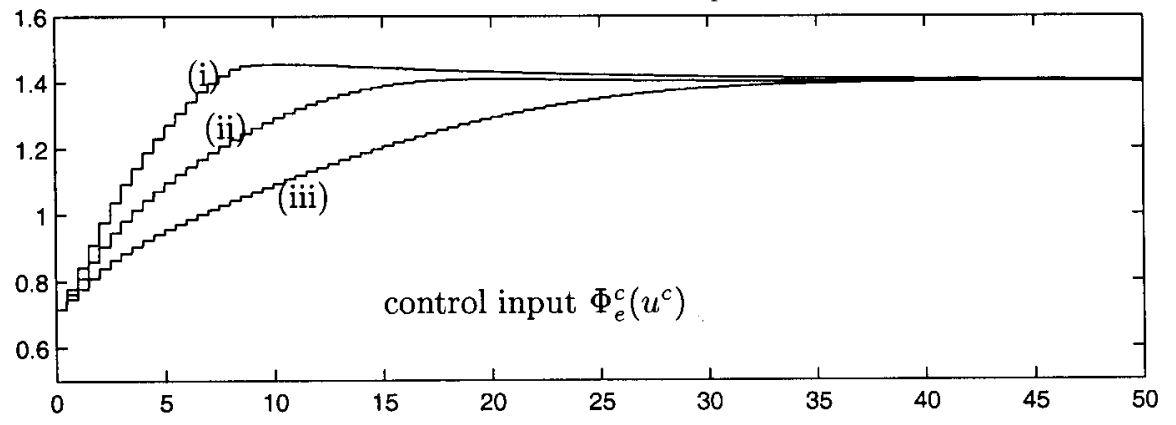

FIG. 9. Control input.

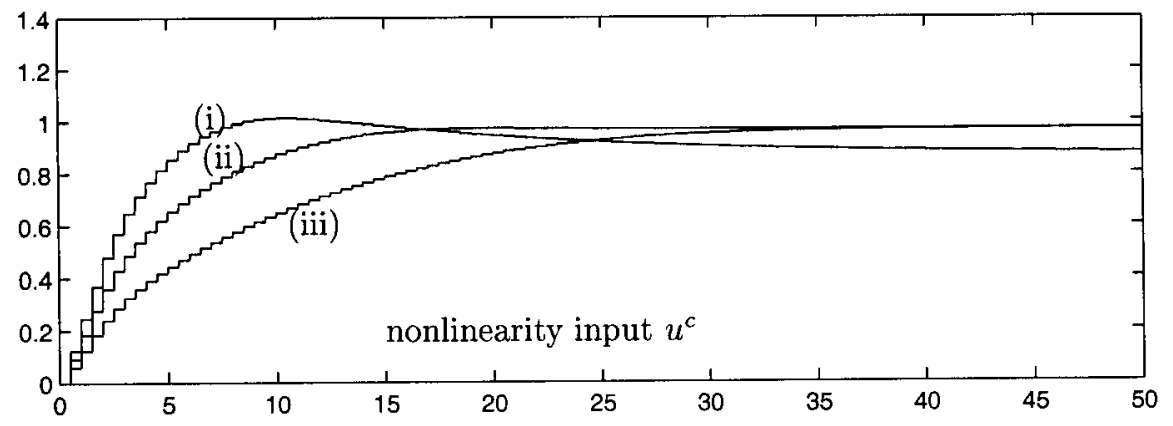

FIG. 10. Input of relay hysteresis nonlinearity.

the corresponding step-response is nondecreasing, and therefore we may apply a result by Özdemir and Townley [23] (see Remark 3.7 in [23]) to obtain the following lower bound for $K$ :

$$
K \geq \frac{1}{\left|\left(\mathbf{G}^{c}\right)^{\prime}(0)\right| / \tau+3 \mathbf{G}^{c}(0) / 2}=: K_{L}
$$

A simple calculation yields that

$$
\mathbf{G}^{c}(0)=\frac{x_{1}\left(1-x_{2}\right)}{\kappa}, \quad\left(\mathbf{G}^{c}\right)^{\prime}(0)=-\frac{x_{1}\left(1-x_{2}\right)\left(6 T \kappa+1-\varepsilon^{2}-x_{1}^{2}-\left(1-x_{2}\right)^{2}\right)}{6 \kappa^{2}},
$$




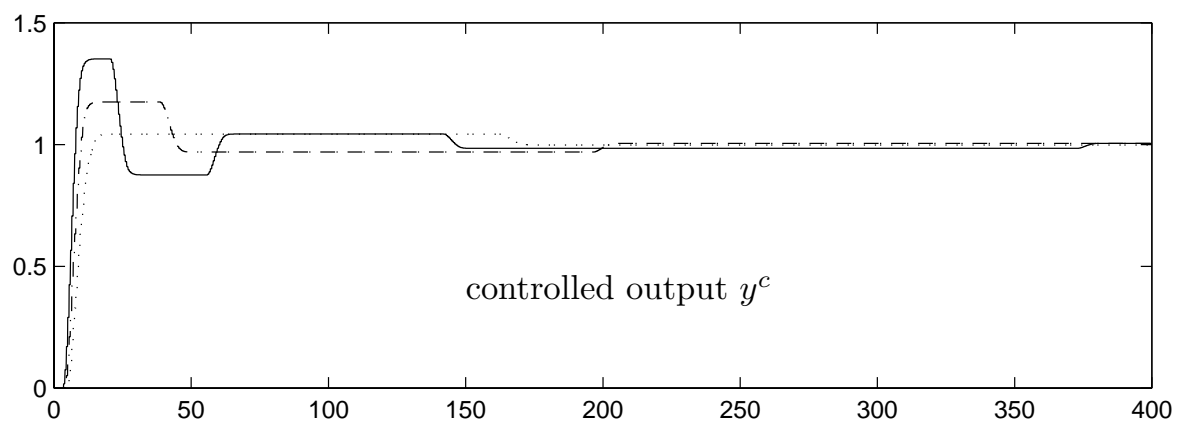

FIG. 11. Controlled output.

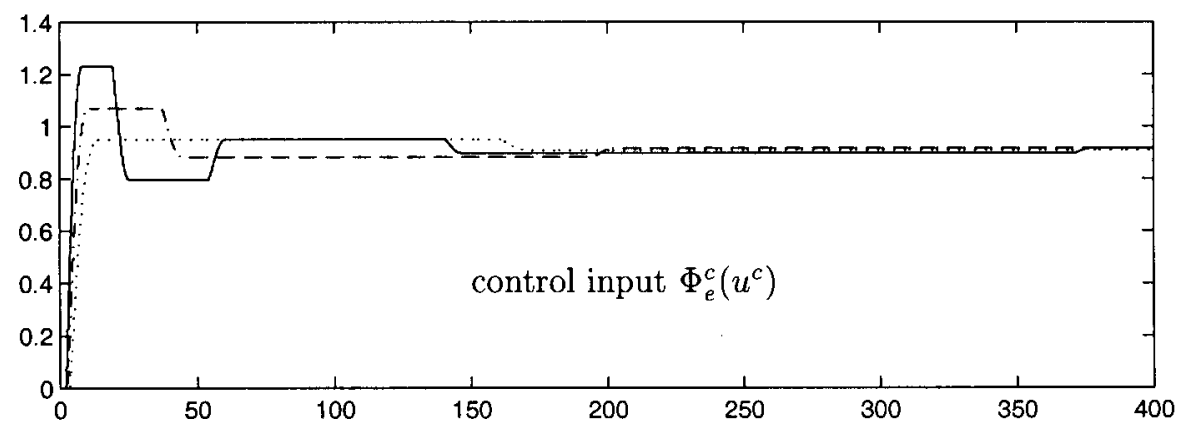

FIG. 12. Control input.

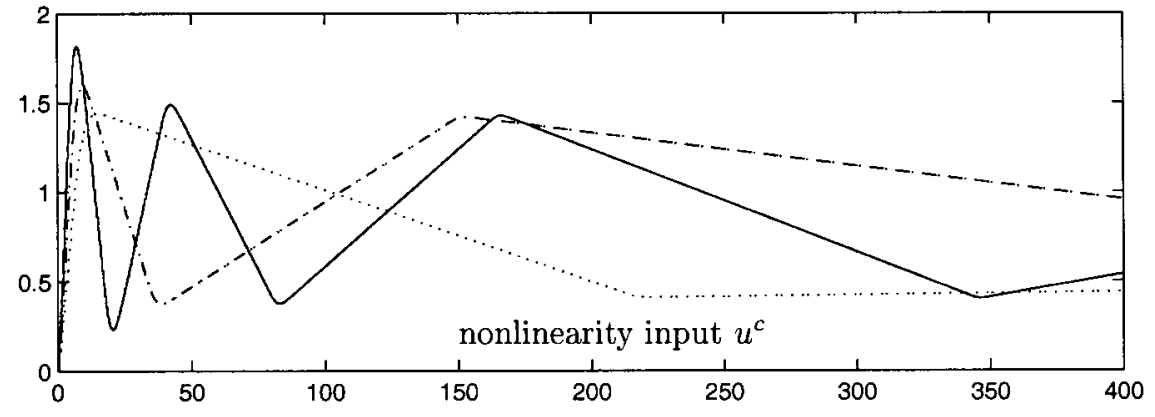

FIG. 13. Input of backlash hysteresis nonlinearity.

and therefore, using (6.1),

$$
K \geq K_{L}=\frac{6 \kappa^{2} \tau}{x_{1}\left(1-x_{2}\right)\left(6 T \kappa+1-\varepsilon^{2}-x_{1}^{2}-\left(1-x_{2}\right)^{2}+9 \kappa \tau\right)}
$$

By Theorem 5.3, for all $k \in\left(0, K_{L} / \lambda\right) \subset(0, K / \lambda)$, the sampled-data control (5.10) guarantees asymptotic tracking of all reference values $r$ which are feasible in the sense that $r / \mathbf{G}^{c}(0) \in \operatorname{clos}\left(\operatorname{NVS} \Phi^{c}\right)$. For purposes of illustration, we adopt the following values:

$$
\kappa=0.1, \quad x_{1}=\frac{1}{3}, \quad x_{2}=\frac{2}{3}, \quad T=1, \quad \tau=0.5, \quad \varepsilon=0.01 .
$$

For these specific values we obtain $K_{L} \approx 0.147$. 
We consider relay and backlash hysteresis operators:

(a) Let $\Phi^{c}=\mathcal{R}_{0}$ be a relay hysteresis operator, where $a_{1}=-1, a_{2}=1, \rho_{1}(v)=$ $\sqrt{v+1.1}$, and $\rho_{2}(v)=\sqrt{0.1}+\sqrt{2.1}-\sqrt{1.1-v}$ (see section 4 for the definition and [11] for more details). Then $\Phi^{c} \in \mathcal{C}(1.6)$, and $\operatorname{NVS} \Phi^{c}=\operatorname{im} \rho_{1} \cup \operatorname{im} \rho_{2}=\mathbb{R}$. For reference value $r=1.54$

$$
\tilde{r}^{c}=\frac{r}{\mathbf{G}^{c}(0)}=\frac{r \kappa}{x_{1}\left(1-x_{2}\right)}=1.386 \in \operatorname{int}\left(\operatorname{NVS}^{c}\right)
$$

In each of the following three cases of admissible controller gain,

$$
\text { (i) } k=0.08, \quad \text { (ii) } k=0.06, \quad \text { (iii) } k=0.04,
$$

Figure 8 depicts the output behavior of the system under integral control, Figure 9 depicts the corresponding control input, and Figure 10 shows the input $u^{c}$ of the relay nonlinearity. We see from Figure 10 that for (i), $\lim _{t \rightarrow \infty} u^{c}(t)=\rho_{1}^{-1}\left(\Phi_{r}^{c}\right)$, and for (ii) and (iii), $\lim _{t \rightarrow \infty} u^{c}(t)=\rho_{2}^{-1}\left(\Phi_{r}^{c}\right)$.

(b) Let $\Phi^{c}=\mathcal{B}_{0.5,0}$ be a backlash hysteresis operator (see section 4 for the definition and [11] for more details). Then $\Phi^{c} \in \mathcal{C}(1)$, and NVS $\Phi^{c}=\mathbb{R}$. For reference value $r=1$

$$
\tilde{r}^{c}=\frac{r}{\mathbf{G}^{c}(0)}=\frac{r \kappa}{x_{1}\left(1-x_{2}\right)}=0.9 \in \operatorname{int}\left(\operatorname{NVS} \Phi^{c}\right) .
$$

In each of the following three cases of admissible controller gain,

(i) $k=0.145$ (solid line), (ii) $k=0.11$ (dashdot line), (iii) $k=0.08$ (dotted line),

Figure 11 depicts the output behavior of the system under sampled-data control, Figure 12 depicts the corresponding control input, and Figure 13 shows the input $u^{c}$ of the backlash nonlinearity. We remark that the convergence of $u^{c}(t)$ as $t \rightarrow \infty$ is not guaranteed by Theorem 5.5, and in fact it seems that $u^{c}$ does not converge in all three cases.

Figures 8-13 were generated using SIMULINK Simulation Software within MATLAB, wherein a truncated eigenfunction expansion, of order 10, was adopted to model the diffusion process.

Appendix. The infinite-dimensional discrete-time positive-real lemma. The following result is a version of the discrete-time infinite-dimensional positive-real lemma.

Lemma A.1. For a real Hilbert space $X$, let $A \in L(X), B \in L(\mathbb{R}, X), C \in$ $L(X, \mathbb{R})$, and $D \in \mathbb{R}$, and set $\mathbf{G}(z):=C(z I-A)^{-1} B+D$. Assume that $A$ is powerstable and

$$
\operatorname{Re} \mathbf{G}\left(e^{i \theta}\right)>0 \quad \forall \theta \in[0,2 \pi) .
$$

Then there exist $P \in L(X), P=P^{*} \geq 0, L \in L(\mathbb{R}, X)$, and $W \in \mathbb{R}$ such that

$$
\begin{aligned}
A^{*} P A-P & =-L L^{*}, \\
A^{*} P B & =C^{*}-W L, \\
W^{2} & =2 D-B^{*} P B .
\end{aligned}
$$

Although Lemma A.1 should be well known, we were not able to locate it in the literature. Lemma A.1 can be obtained from Lemma A.2 (an infinite-dimensional 
version of the continuous-time positive-real lemma stated below) combined with standard fractional transformation techniques (as used in [6] for the finite-dimensional case). For the sake of brevity, we omit the lengthy but straightforward details, which can be found in [21].

Lemma A.2. For a real Hilbert space $X$, let $A^{c} \in L(X), B^{c} \in L(\mathbb{R}, X), C^{c} \in$ $L(X, \mathbb{R})$, and $D^{c} \in \mathbb{R} ;$ let $\sigma\left(A^{c}\right)$ denote the spectrum of $A^{c}$, and set $\mathbf{G}^{c}(s):=C^{c}(s I-$ $\left.A^{c}\right)^{-1} B^{c}+D^{c}$. Assume that $\sigma\left(A^{c}\right) \subset\{s \in \mathbb{C} \mid \operatorname{Re} s<0\}$ and

$$
\operatorname{Re} \mathbf{G}^{c}(i \omega)>0 \quad \forall \omega \in \mathbb{R} \cup\{ \pm \infty\} .
$$

Then there exist $P^{c} \in L(X), P^{c}=\left(P^{c}\right)^{*} \geq 0, L^{c} \in L(\mathbb{R}, X)$, and $W^{c}>0$ such that

$$
\begin{aligned}
P^{c} A^{c}+\left(A^{c}\right)^{*} P^{c} & =-L^{c}\left(L^{c}\right)^{*}, \\
P^{c} B^{c} & =\left(C^{c}\right)^{*}-W^{c} L^{c}, \\
2 D^{c} & =\left(W^{c}\right)^{2} .
\end{aligned}
$$

In a different form, Lemma A.2 is due to Yakubovich [30] (see also Wexler [29]). For completeness, we include a proof which is based on the positive-real Riccati equation theory developed in van Keulen [7].

Proof of Lemma A.2. By (A.1) we have that $D^{c}>0$; defining $W^{c}:=\sqrt{2 D^{c}}$ gives (A.2c). Furthermore, again by (A.1), it follows from [7] (see Theorem 3.10 and Remark 3.14 in [7]) that there exists $Q^{c} \in L(X), Q^{c}=\left(Q^{c}\right)^{*}$, such that

$$
Q^{c} A^{c}+\left(A^{c}\right)^{*} Q^{c}=\left(1 / W^{c}\right)^{2}\left(\left(B^{c}\right)^{*} Q^{c}+C^{c}\right)^{*}\left(\left(B^{c}\right)^{*} Q^{c}+C^{c}\right) .
$$

Setting

$$
P^{c}:=-Q^{c}, \quad L^{c}:=\left(1 / W^{c}\right)\left(C^{c}-\left(B^{c}\right)^{*} P^{c}\right)^{*}
$$

yields (A.2a) and (A.2b). Since $\sigma\left(A^{c}\right) \subset\{s \in \mathbb{C} \mid \operatorname{Re} s<0\}$, it follows from (A.2a) by a routine argument that $P^{c} \geq 0$.

\section{REFERENCES}

[1] M. Brokate, Hysteresis operators, in Phase Transitions and Hysteresis, A. Visintin, ed., Springer-Verlag, Berlin, 1994, pp. 1-38.

[2] M. Brokate and J. Sprekels, Hysteresis and Phase Transitions, Springer-Verlag, New York, 1996.

[3] G. W. M. Coppus, S. L. Sha, And R. K. Wood, Robust multivariable control of a binary distillation column, IEE Proc., Part D, 130 (1983), pp. 201-208.

[4] E. J. DAvison, Multivariable tuning regulators: The feedforward and robust control of a general servomechanism problem, IEEE Trans. Automat. Control, 21 (1976), pp. 35-47.

[5] G. Gripenberg, S.-O. Londen, and O. Staffans, Volterra Integral and Functional Equations, Cambridge University Press, Cambridge, UK, 1990.

[6] L. Hitz And B. D. O. Anderson, Discrete positive-real functions and their application to system stability, Proc. IEE, 116 (1969), pp. 153-155.

[7] B. A. M. van Keulen, $H^{\infty}$-Control for Infinite-Dimensional Systems: A State-Space Approach, Birkhäuser Boston, Boston, 1993.

[8] M. A. Krasnosel'skiI And A. V. Pokrovskil̆, Systems with Hysteresis, Springer-Verlag, Berlin, 1989.

[9] H. Logemann AND R. F. CuRTain, Absolute stability results for well-posed infinite-dimensional systems with applications to low-gain integral control, ESAIM Control Optim. Calc. Var., 5 (2000), pp. 395-424.

[10] H. Logemann and A. D. Mawby, Extending Hysteresis Operators to Spaces of Piecewise Continuous Functions, Mathematics Preprint 00/14, University of Bath, Bath, UK, 2000; also available online from http://www.maths.bath.ac.uk/MATHEMATICS/preprints.html and via anonymous ftp from ftp.maths.bath.ac.uk from the directory pub/preprints. 
[11] H. Logemann And A. D. MAwBy, Low-gain integral control of infinite-dimensional regular linear systems subject to input hysteresis, in Advances in Mathematical Systems Theory, F. Colonius et al., eds., Birkhäuser Boston, Boston, 2001, pp. 255-293.

[12] H. Logemann And E. P. Ryan, Time-varying and adaptive discrete-time low-gain control of infinite-dimensional linear systems with input nonlinearities, Math. Control Signals Systems, 13 (2000), pp. 293-317.

[13] H. Logemann And E. P. Ryan, Time-varying and adaptive integral control of infinitedimensional regular linear systems with input nonlinearities, SIAM J. Control Optim., 38 (2000), pp. 1120-1144.

[14] H. Logemann, E. P. RYAn, AND S. TOWnley, Integral control of linear systems with actuator nonlinearities: Lower bounds for the maximal regulating gain, IEEE Trans. Automat. Control, 44 (1999), pp. 1315-1319.

[15] H. Logemann, E. P. Ryan, And S. Townley, Integral control of infinite-dimensional linear systems subject to input saturation, SIAM J. Control Optim., 36 (1998), pp. 1940-1961.

[16] H. Logemann And S. Townley, Discrete-time low-gain control of uncertain infinitedimensional systems, IEEE Trans. Automat. Control, 42 (1997), pp. 22-37.

[17] H. LOgEmann And S. TOWnLey, Low-gain control of uncertain regular linear systems, SIAM J. Control Optim., 35 (1997), pp. 78-116.

[18] J. Lunze, Robust Multivariable Feedback Control, Prentice Hall, London, 1988.

[19] J. Lunze, Experimentelle Erprobung einer Einstellregel für PI-Mehrgrößenregler bei der Herstellung von Ammoniumnitrat-Harnstoff-Lösung, Messen Steuern Regeln, 30 (1987), pp. $2-6$.

[20] J. W. Macki, P. Nistri, And P. Zecca, Mathematical models for hysteresis, SIAM Rev., 35 (1993), pp. 94-123.

[21] A. D. MaWBy, Integral Control of Infinite-Dimensional Linear Systems Subject to Input Hysteresis, Ph.D. thesis, Department of Mathematical Sciences, University of Bath, Bath, UK, 2000.

[22] M. Morari, Robust stability of systems with integral control, IEEE Trans. Automat. Control, 30 (1985), pp. 574-577.

[23] N. Özdemir And S. Townley, Robust Sampled-Data Integral Control by Variable and Adaptive Sampling, Preprint, School of Mathematical Sciences, University of Exeter, Exeter, UK, 2000.

[24] A. PAZY, Semigroups of Linear Operators and Applications to Partial Differential Equations, Springer-Verlag, New York, 1983.

[25] G. WeIss, Transfer functions of regular linear systems. I. Characterization of regularity, Trans. Amer. Math. Soc., 342 (1994), pp. 827-854.

[26] G. WEISS, Admissibility of unbounded control operators, SIAM J. Control Optim., 27 (1989), pp. $527-545$.

[27] G. WEISs, Admissible observation operators for linear semigroups, Israel J. Math., 65 (1989), pp. $17-43$.

[28] G. WEISS, The representation of regular linear systems on Hilbert spaces, in Distributed Parameter System, F. Kappel, K. Kunisch, and W. Schappacher, eds., Birkhäuser Verlag, Basel, 1989, pp. 401-416.

[29] D. WeXler, On frequency domain stability for evolution equations in Hilbert spaces via the algebraic Riccati equation, SIAM J. Math. Anal., 11 (1980), pp. 969-983.

[30] V. A. Yakubovich, A frequency domain theorem for the case in which the state and control spaces are Hilbert spaces with an application to some problems in the synthesis of optimal controls I, Siberian Math. J., 15 (1974), pp. 457-476. 